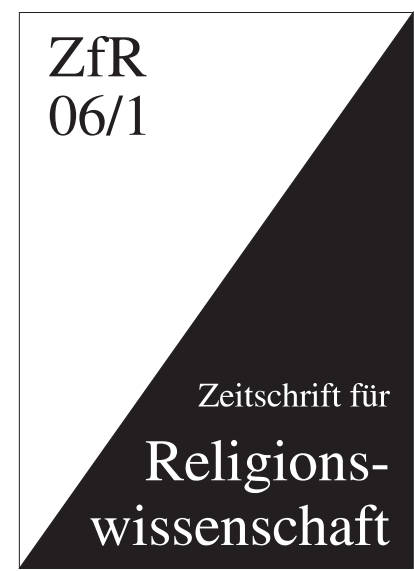

Herausgegeben im Auftrag der Deutschen Vereinigung für Religionswissenschaft von

Christoph Auffarth

Max Deeg

Manfred Hutter

Hubert Knoblauch

Jörg Rüpke

Begründet 1993 von

Burkhard Gladigow

Monika Horstmann

Günter Kehrer

Kurt Rudolph

Hubert Seiwert

Schriftleitung:

Prof. Dr. Christoph Auffarth Universität Bremen

Fachbereich 9

Religionswissenschaft /

Religionspädagogik

Badgasteiner Str. 1

D-28359 Bremen

Bezugspreise und Verlag:

Einzelheft: 26,00 $€$.

Jahresabonnement: 47,00 €.

Jahresabonnement für

Studierende: $28,00 €$.

Jeweils zzgl. Porto.

Die ZfR erscheint zweimal im Jahr.

diagonal-Verlag

Alte Kasseler Str. 43

D-35039 Marburg

ISSN 0943-8610

\section{ZfR}

\section{Zeitschrift für}

Religionswissenschaft

\section{Jahrgang 2006}

\section{Inhalt}

\section{Beiträge}

\section{Anne Koch:}

»Religionshybride« Gegenwart.

Religionswissenschaftliche Analyse

anhand des Harry-Potter-Phänomens

\section{Karsten Lehmann:}

Institutionen religiöser Pluralität.

Vergleichende Analyse der christlichen und muslimischen Migrantengemeinden in Frankfurt am Main

\section{Heinrich Schäfer:}

»Die« Pfingstbewegung in Lateinamerika...?

Zur Untersuchung des Verhältnisses zwischen

religiöser Praxis und gesellschaftlichen

Strukturen

Buchbesprechungen 


\title{
Heinrich Schäfer
}

\section{$»$ Die « Pfingstbewegung in Lateinamerika...?}

\author{
Zur Untersuchung des Verhältnisses zwischen \\ religiöser Praxis und gesellschaftlichen Strukturen
}

Inhalt

Religiöse Bewegungen sind keine homogenen Einheiten, auch wenn sie - wie »die" Pfingstbewegung - häufig als solche behandelt werden. Begriffsbildung ist natürlich unumgänglich. Gleichwohl ist es interessant, die innere Differenzierung von religiösen Bewegungen zu untersuchen und deren Gründe zu eruieren. Dazu braucht es ein wissenschaftliches Instrumentarium, welches erlaubt, gesellschaftliche Bedingungen zu religiöser Praxis - besonders zu kognitiven Dispositionenin Beziehung zu setzen. Am Beispiel der Pfingstbewegung im Guatemala der achtziger Jahre sowie auf dem Hintergrund bourdieuscher Sozialtheorie wird ein solches Instrumentarium skizziert, eine starke Ausdifferenzierung innerhalb der Pfingstbewegung aufgezeigt, und die darin wirksamen nicht-religiösen Faktoren werden sichtbar gemacht.

Wenn... die Art der erstrebten Heilsgüter stark beeinflusst war durch die Art der äußeren Interessenlage... und also durch die soziale Schichtung selbst, so war umgekehrt auch die Richtung der ganzen Lebensführung... auf das Tiefgreifendste bestimmt durch... religiös bedingte Wertungen und Stellungnahmen. ${ }^{1}$

» $\mathrm{D}_{\mathrm{ie}}$ « Pfingstbewegung ist - wie alle anderen religiösen Bewegungen auch - keine homogene Einheit. Im Lauf der letzten Jahrzehnte hat sich diese Einsicht auch bei der empirischen Forschung über die Bewegung in Lateinamerika herauskristallisiert. ${ }^{2}$ Im vorliegenden Aufsatz möchte ich der Frage nachgehen, wie weit die festgestellte Uneinheitlichkeit ${ }^{3}$ auf spezifische unterschiedliche Erfahrungen der

1 M. Weber, Gesammelte Aufsätze zur Religionssoziologie, Bd. 1, Tübingen 1988, 259.

2 Vgl. exemplarisch D. Stoll, Is Latin America Turning Protestant?, Berkeley 1990; D. Martin, Tongues of Fire, Oxford 1990; H. Schäfer, Protestantismus in Zentralamerika, Frankfurt 1992, 90; ders., » $>$ Herr des Himmels, gib uns Macht auf der Erde!««, in: Materialdienst des Konfessionskundlichen Instituts 3, Bensheim 1992, 43-48.

3 ... oder gar »Ambiguität«, D. Martin, Pentecostalism, Oxford 2002, 83. 
Akteure etwa mit gesellschaftlicher Deklassierung, relativer Deprivation, Verhinderung sozialen Aufstiegs etc. zurückgehen. Im weiteren religionssoziologischen Diskussionszusammenhang ist damit die - gewissermaßen klassische - Frage thematisch, welches Verhältnis man zwischen sozialstrukturellen Positionen und religiösen Dispositionen des Wahrnehmens, Urteilens und Handelns annehmen und wie man es untersuchen kann. Ich erörtere diese Frage hier anhand der Ergebnisse einer empirischen Studie im Bürgerkrieg Guatemalas in den achtziger Jahren. Dieses Feld bot sozusagen »Laborbedingungen« für die genannte Fragestellung, denn die Gesellschaft war sozial, politisch, ökonomisch und kulturell extrem polarisiert. ${ }^{4}$ Ich werde zeigen, dass unter diesen Umständen aus einem gemeinsamen »pfingstlichen « Symbolinventar zwei vollkommen unterschiedliche religiöse Praxisformen entstanden sind, und ein Verfahren skizzieren, wie man den Zusammenhang zwischen gesellschaftlichen Strukturen und religiöser Praxis nachzeichnen kann. Dabei konzentriere ich mich auf zwei Fragen:

- Wie lässt sich das Verhältnis von religiösem Feld und gesamtgesellschaftlichen Bedingungen konstruieren?

- Wie kann man das Verhältnis zwischen gesellschaftlichen Bedingungen und kognitiven religiösen Dispositionen besser verstehen?

Ich knüpfe mit den folgenden Überlegungen an die jüngere Forschung zu sozialen Bewegungen sowie an die Soziologie Bourdieus an.5 Aus der Bewegungstheorie werden die Theoreme einfließen, dass soziale Bewegungen sich normalerweise in Reaktion auf Krisenerfahrungen hin mobilisieren, und dass zur Erfassung dieser Dynamik die Rekonstruktion von »cognitive maps « ${ }^{6}$ nützlich ist. Diese Anregungen bette ich in den Rahmen von Bourdieus Sozialtheorie ein, unter besonderer Berücksichtigung der Begriffe des sozialen Raumes, der Felder und der praktischen Logik; letzteres Konzept wird leicht weiter entwickelt. ${ }^{7}$ Dieser Ansatz ermöglicht es, ein Modell zu konstruieren, in dem gesellschaftliche Positionen mit religiösen Dispositionen in Beziehung gesetzt und diese Beziehungen analysiert werden können.

In diesem Rahmen arbeite ich im Folgenden mit Teilen der Ergebnisse einer empirischen Untersuchung über religiöse Bewegungen in Guatemala und Nicaragua in der Bürgerkriegszeit (1985 und 1986) sowie einer kurzen Nachuntersuchung (Guatemala 1993). Die Feldforschung erfolgte unter der Fragestellung, welche Effekte die Bürgerkriegssituation in Guatemala und Nicaragua auf religiöse Bewegun-

4 Ich kann hier aus Raumgründen keine Einführung in die historischen Umstände geben. Vgl. aber H. Schäfer, Protestantismus in Zentralamerika...

5 Näheres zur Theoriediskussion in diesem Artikel musste ich aus Raumgründen streichen. Vgl. aber H. Schäfer, Zur Theorie von kollektiver Identität und Habitus am Beispiel sozialer Bewegungen, Berlin 2002 (Diss., Mikrofiche); ders., Praxis - Theologie - Religion, Frankfurt 2004; ders., »Identität als Netzwerk «, in: Berliner Journal für Soziologie 2, 2005, 261 284 (Corrigendum auf www.homes.uni-bielefeld.de/hschaefer3/index.html).

6 H. Kitschelt, »Resource Mobilization Theory. A critique«, in: D. Rucht (Hg.), Research on social movements, Frankfurt 1991, 323-347, hier: 332.

7 Dadurch werden u. a. die »kognitiven Landkarten« zu praxeologischen; es handelt sich also hier nicht um eine »semiologische« Untersuchung. 
gen und insbesondere die Pfingstbewegung hat. ${ }^{8}$ Aus dem Gesamtsample ${ }^{9}$ wurden für die hier zugrunde liegende Untersuchung kleinere Stichproben aus Guatemala ausgewählt. ${ }^{10}$ Alle Stichproben der Feldforschung wurden durch eine Kombination von theoretischer Vorauskonstruktion des Feldes und theoretical sampling ermittelt und strukturiert. Sie ließen sich nach demographischen Kriterien (Berufe, Einkommen, Bildung, Wohnverhältnisse, Wohnort) verschiedenen Positionen des gesellschaftlichen Raumes zuordnen. Für die hier diskutierte Auswahl wurden zusätzlich zu den Leitfadeninterviews pro Habitusformation 10 Predigten (»Reden «) mitgeschnitten, weitere Expertengespräche geführt sowie teilnehmende Beobachtung in Gottesdiensten und im Alltagshandeln durchgeführt. Die Nachuntersuchung erfolgte 1993 gezielt an einem Ort der Feldforschung von 1985/86 und hatte das veränderte gesellschaftliche Engagement der früheren Interviewpartner zum Gegenstand von Gesprächen und teilnehmender Beobachtung. Die Analyse des Materials erfolgte mit der an Greimas angelehnten Methode zur Untersuchung praxeologischer Operatoren, die unten (Kap. 2) knapp skizziert wird. Die Untersuchung repräsentiert also keine Selbstbeschreibungen der Akteure, sondern rekonstruiert objektive »Tiefenstrukturen « (Greimas) des sozialen Sinnes von Akteuren. ${ }^{11}$ Diese liegen - sozusagen - »oberhalb« des Unbewussten und »unterhalb« des Bewusstseins der Akteure. ${ }^{12}$

Um die o. g. Forschungsfragen zu beantworten, werde ich zwei Modelle konstruieren. Sie sollen Folgendes leisten. Das Modell der Logik der Praxis (in Kap. 1) soll verdeutlichen:

- die Stellung unterschiedlicher gesellschaftlicher Positionen zueinander gemäß differenzieller Kapitalverteilung, inkl. Laufbahnperspektiven einzelner Positionen und Konfliktlinien zwischen ihnen (sozialer Raum);

- das Verhältnis von den Positionen, die die untersuchten Akteure im religiösen Feld einnehmen, zu den Positionen des gesellschaftlichen Raumes.

Damit wäre das religiöse Feld in einer Weise konstruiert, die es durchsichtig werden lässt auf die gesellschaftliche Kapitalstärke der kollektiven religiösen Akteure. Im Blick auf die praktische Logik modelliere ich (in Kap. 2) den Prozess, in dem die Akteure ihre positionsspezifische Erfahrung vermittels Wahrnehmung, Urteil und Handeln religiös verarbeiten. Am empirischen Material wird dazu das

8 Die Ergebnisse der empirischen Untersuchung sollten zu Beginn der neunziger Jahre als Dissertation im Fach Ev. Theologie veröffentlicht werden, wurden aber als »zu soziologisch" schon im Vorfeld nicht zugelassen. Das 600-seitige Manuskript wurde - vor allem aufgrund meiner späteren Beschäftigung in Lateinamerika - bisher nicht veröffentlicht. Eine stark komprimierte Fassung findet sich in H. Schäfer, Zur Theorie von kollektiver Identität... Die Kontrollgruppe ist Gegenstand des zweiten Teils von H. Schäfer, Protestantismus in Zentralamerika...

9 In Guatemala und Nicaragua habe ich in zwei Jahren insgesamt 195 Interviews geführt und 112 Predigten mitgeschnitten.

1020 Personen aus der Habitusformation der NPERG und 18 der PERG/U.

11 ... ähnlich den »latenten Sinnstrukturen« bei U. Oevermann, »Die Struktur sozialer Deutungsmuster - Versuch einer Aktualisierung «, in: Sozialer Sinn 1, 2001, 35-81.

12 H. Schäfer, Zur Theorie von kollektiver Identität...; ders., »Identität als Netzwerk...«. 
Modell eines Netzwerks praktisch-logischer Operatoren entwickelt. Damit sollte Folgendes modelliert werden können:

- der Ansatz des kognitiven Prozesses bei der Krisenerfahrung;

- die Transformation von Erfahrung über religiös bestimmte Wahrnehmung, Urteil und Handlungsentwürfe;

- die Systematizität praktischer Logik als Netzwerk bedeutungsbildender religiöser Relationen;

- die Übertragung praktischer Schemata zwischen dem religiösen und anderen Praxisfeldern;

- der vielfältige Feldbezug der praktischen Logik religiösen Handelns.

Damit wären die spezifischen Operationen praktischer Logik der religiösen Bewegungen aufgeschlüsselt.

Der letzte Schritt ist ein Vergleich zwischen praktischer Logik und Logik der Praxis (Kap. 3). Das ist erst vom Modell der praktischen Logik her möglich, denn erst dieses macht erkennbar, welche Muster überhaupt zu vergleichen sind. Der Vergleich soll dartun,

- ob die unterschiedlichen sozialen Positionen unterschiedliche religiöse Dispositionen aus demselben Zeicheninventar geformt haben;

- ob sich die gesellschaftlichen Konfliktlinien und die positionsspezifischen Laufbahnperspektiven auf die religiösen Wahrnehmungs-, Urteils- und Handlungslogiken niederschlagen;

- ob und wie sich die positionsspezifischen Krisenerfahrungen auf die Logik religiöser Praxis auswirken;

- ob Homologien zwischen den Schemata der praktischen Logiken religiöser Akteure und den für sie relevanten Ablaufschemata des gesellschaftlichen Funktionssystems insgesamt (Logik der Praxis) bestehen.

Damit wären dann unsere Leitfragen beantwortet.

\section{Logik der Praxis: Sozialer Raum und religiöses Feld}

Zur Rekonstruktion der Logik der Praxis werden zunächst die wichtigsten sozialstrukturellen Positionen der guatemaltekischen Gesellschaft bestimmt. Dann wird das religiöse Feld auf dem Modell abgetragen.

\subsection{Sozialer Raum}

Die methodische Operationalisierung des Raumbegriffs erfolgt auf der Basis von gesellschaftlichen Strukturdaten durch ein Korrelationsmodell, orientiert an Ge- 
samtkapitalvolumen und Kapitalstruktur (Abb. 1).13 Auf der senkrechten Achse eines Koordinatensystems wird die generelle Kapitalstärke (alle Kapitalformen wie ökonomisches, politisches etc.) von Raumpositionen (d. h. konkret: kollektiven Akteuren) abgetragen. Auf der x-Achse wird speziell das kulturelle Kapital abgetragen. Dieses ist definiert als im Prozess der Modernisierung verwertbares Wissen. Das Modell erlaubt die Konstruktion einer (arbeitsteilig) differenzierten Gesellschaft unter gleichzeitiger Beachtung sozialer Ungleichheit. Zudem können positionsspezifische Laufbahnperspektiven und gesellschaftliche Konfliktlinien berücksichtigt werden.

Das Modell des gesellschaftlichen Raumes in Guatemala (vgl. Abb. 1) zeigt im oberen Drittel (aber mit schlechten Laufbahnperspektiven) die Oligarchie (Latifundien) und die »alten « Militärs (faschistoid) im traditionellen Sektor (kulturelles Kapital). Die neue Bourgeoisie (innovative Technologie, neoliberal-demokratisch, US-amerikanische Bildungstitel), technokratische »junge Militärs «(Westpoint) sowie gut situierte Freiberufler und gehobenes Management im modernisierenden Sektor haben positive, aber durch die Krise blockierte Laufbahnperspektiven. Im mittleren Drittel auf der modernen Seite liegen das neue Kleinbürgertum (Anwälte, Ärzte, Ingenieure etc.) und das exekutive Kleinbürgertum (Verwaltungsbeamte, Hochschullehrer etc.); auf der traditionellen Seite liegt das alte Kleinbürgertum, inkl. der indianischen Mittelschicht (mittlere Händler, Gewerbetreibende etc.). Das untere Drittel umfasst den größten Teil der indigenen und mestizischen Bevölkerung. Die traditionelle Unterschicht (kleine Ladenbesitzer, Handwerker etc.) erfährt Modernisierung und Krise als sozialen Abstieg. Proletarisierte und semi-proletarisierte Arbeitskräfte sehen modernisierungsbedingte Aufstiegschancen durch Krise und Konflikte blockiert. Die Marginalisierten und aus der formellen Ökonomie Ausgeschlossenen (»Müllmenschen« etc.) sind indifferent.

Die Gesamtlage ist durch zwei Prozesse charakterisiert: eine selektive und prekär verlaufende Modernisierung sowie eine Krise in ökonomischer, politischer und militärischer Dimension mit dem Resultat eines Bürgerkrieges. Es lassen sich zwei für uns relevante Konfliktlinien bestimmen: die erste zwischen herrschenden und subordinierten gesellschaftlichen Positionen (Konflikt A) und die zweite, in unterschiedlichen gesellschaftlichen Schichten, zwischen modernisierenden und traditionellen Positionen (Konflikte B1 und B2).

Gesamtlage, positionsspezifische Laufbahnperspektiven und gesellschaftliche Konfliktlinien sind Ausgangsbedingungen für die Mobilisierung sozialer Bewegungen, von Guerilla und Paramilitärs bis hin zu religiösen Bewegungen. Die gesellschaftlichen Konflikte schlagen auf die Strukturierung der verschiedenen gesellschaftlichen Praxisfelder durch. Unter anderem wird die Krise religiös verarbeitet.

13 Vgl. P. Bourdieu, Die feinen Unterschiede, Frankfurt 1987, 212; K. Eder, »Klassentheorie als Gesellschaftstheorie«, in: ders. (Hg.), Klassenlage, Lebensstil und kulturelle Praxis, Frankfurt 1989, 15-43, hier: 33. 


\subsection{Religiöses Feld}

Im Folgenden werden die Positionen des religiösen Feldes mit dem Modell des Raumes korreliert. Die religiösen Positionen werden als »Habitusformationen «14 verstanden, die anhand religiöser Geschmacksindikatoren ermittelt und mittels sozialstruktureller Daten der Bewegungsangehörigen im Raum verortet werden.

In Guatemala zeigt sich das religiöse Feld deutlich polarisiert. Die beiden hier untersuchten Bewegungen nehmen entgegengesetzte Positionen ein (Abb. 1).

Mit dem Code »NPERG« (Neopfingstliche etablierte religiöse Gruppierung) bezeichne ich die eine der beiden Habitusformationen. Sie befindet sich in einer homologen Raumposition zum modernisierenden Bürgertum und damit in doppelter gesellschaftlicher Frontstellung: gegen die rebellierenden Klassen »von unten « und gegen die alte Oligarchie (Konflikte A und B1). Im religiösen Feld entsprechen dem, erstens, eine Frontstellung gegenüber den Basisgemeinden (BAS), den unabhängigen Pfingstgruppierungen (PRG) und PERG (der Pfingstlichen etablierten religiösen Gruppierung) sowie, zweitens, gegen den traditionellen Katholizismus (TRAK). Die Laufbahnperspektive der Akteure war bis zur Krise Anfang der achtziger Jahre positiv, wurde dann aber jäh gebremst. Die Akteure verfügen allerdings über genügend Machtressourcen, um die gesellschaftlichen Probleme anzugehen.

Die hier untersuchte Stichprobe aus der Habitusformation PERG ist sozial homogen und liegt im gesellschaftlichen Raum homolog zur traditionellen Unterschicht sowie zu Teilen der Arbeiterschaft und der Marginalisierten. Sie ist eine Untergruppe der PERG, die ich hier PERG/U (wie Unterschicht) nenne. Ihre wichtigste gesellschaftliche Frontstellung ist die zu »denen da oben«. Diesem Gegensatz entsprechen im religiösen Feld latente und manifeste Konflikte mit dem traditionellen Katholizismus (TRAK) und mit NPERG. Die zweitwichtigste Frontlinie verläuft zu den politisch engagierten Proletariern; im religiösen Feld: die politischen Christen der Basisgemeinden (BAS) und Teile der unabhängigen Pfingstgruppen (PRG). Die Laufbahnperspektive der PERG/U-Anhänger, herkömmlich bescheiden, hat sich durch die Krise radikal verschlechtert. Machtressourcen sind nicht vorhanden.

Die anderen Habitusformationen werden hier nur als externer Handlungskontext von PERG/U und NPERG berücksichtigt: PRG (Pfingstliche religiöse Gruppierung) ist tendenziell stärker der (modernisierenden) Lohnarbeiterposition homolog. Ähnlich ist die reformerische bis revolutionäre BAS (Basisgemeinden von Katholiken oder Protestanten, Theologie der Befreiung) in der organisierten Unterschicht und bei sub-kleinbürgerlichen Intellektuellen verortet. Durch die militärische Repres-

14 Unter »Habitusformationen « verstehe ich die objektiven Häufungen bestimmter Dispositionsstrukturen von Akteuren in bestimmten Positionen eines definierten Feldes. »Formation « (statt »Klasse«) assoziiert die Vorstellung von geregelt sich bewegenden Individuen (etwa Fischen in Schwärmen). Habitusformationen können auch als kollektive Identitätsformationen gelesen werden (H. Schäfer, »Identität als Netzwerk...«), wenn die entsprechenden Akteure kollektiv mobilisiert sind. Oevermann verwendet denselben Begriff, aber analog zu »Deutungsmuster« (U. Oevermann, »Die Struktur sozialer Deutungsmuster...«, 45). 


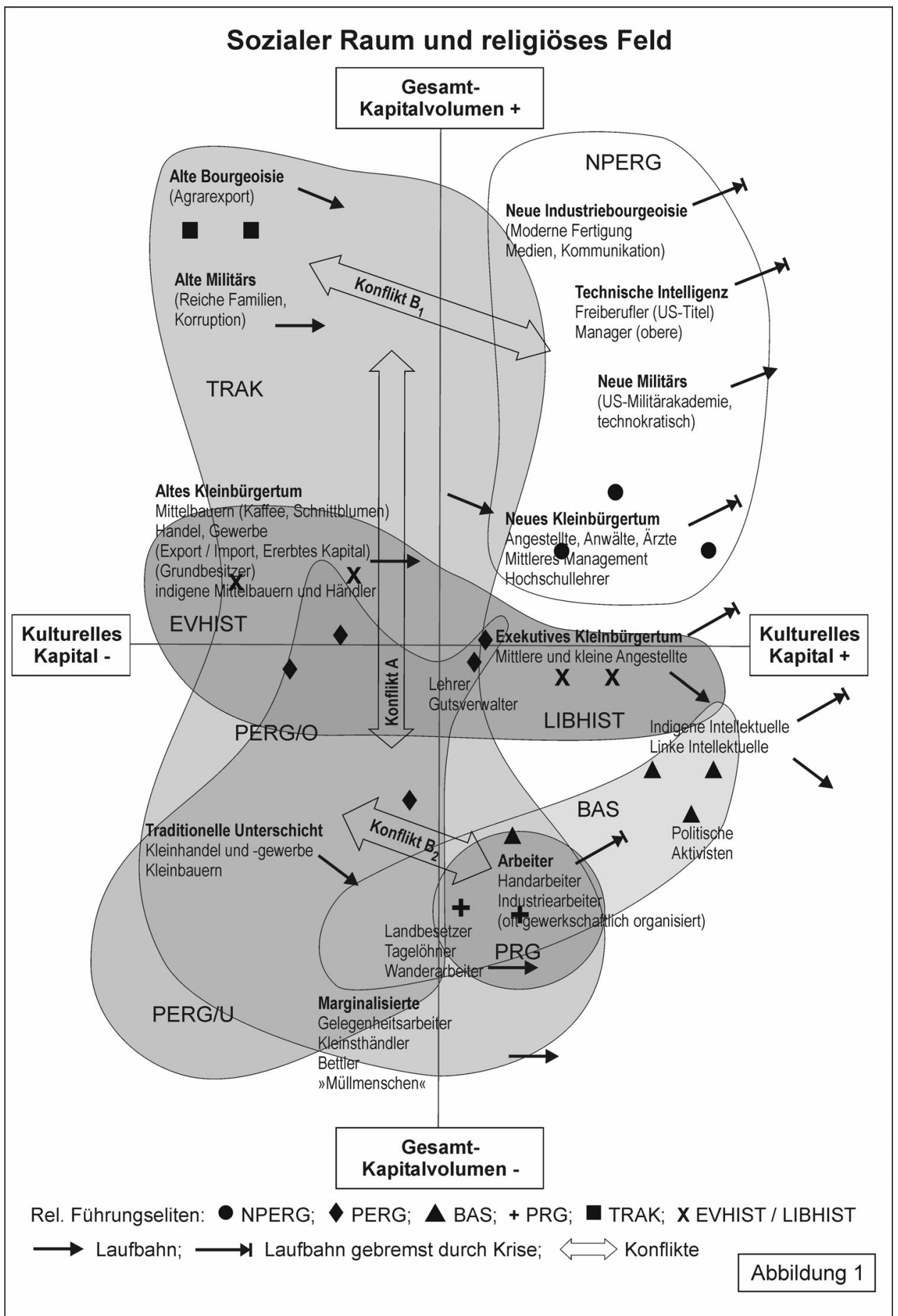

Notiz des Autors (August 2008): Entsprechend einiger grundsätzlicher Entscheidungen im Blick auf die Forschungslogik, neuer Projekte habe ich folgende Veränderungen dieses Modells vorgenommen:

- Auf der horizontalen Achse wird künftig -- wie im Raummodell Bourdieus -- kulturelles und ökonomisches Kapital gegenläufig abgetragen, so dass die entsprechende Kapitalstruktur dargestellt wird;

- Die horizontale Achse wird spiegelverkehrt umgewandelt ("kulturelles Kapital minus" auf der rechten Seite), wodurch die gesamt Darstellung spiegelverkehrt erfolgt;

- Das gesamte Modell wird als ein Modell des "Raumes religiöser Stile" verstanden und von einer vollkommen anderen theoretischen Modellierung des religiösen Feldes kategorial unterschieden. 
sion ist sie sehr geschwächt. TRAK (Traditioneller Katholizismus) verbindet wundergläubigen Volkskatholizismus mit hierarchischer Institution. Sie beerbt die frühere Monopolstellung der katholischen Kirche, ist aber auf ältere gesellschaftliche Positionen reduziert mit dem Machtschwerpunkt auf der alten Oligarchie. EVHIST (Historische Evangelikale, Schrift-Fundamentalismus) liegt homolog zum konservativen Kleinbürgertum, ist auf die Wahrung angestammter Positionen fixiert und rechtsgerichtet. Die LIBHIST-Formation (liberaler Historischer Protestantismus) homolog zum modernen exekutiven Kleinbürgertum - tendiert zur Anpassung an Modernisierungsprozesse und vorsichtigem demokratischen Reformismus.

In diesem religiösen Feld lassen sich in der Krise der frühen achtziger Jahre folgende Dynamiken beobachten. Im Blick auf die Laufbahnperspektiven teilen NPERG und BAS die Erfahrung des Einbruchs hoher Erwartungen, während TRAK und PERG Stagnation und soziales Abrutschen erleben. Entlang der gesellschaftlichen Konfliktlinie zwischen »oben« und »unten« sind TRAK teilweise und NPERG gänzlich »oben « positioniert, PERG/U, PRG und BAS »unten «. Entsprechend unterschiedliche praktische Logiken sind zu vermuten. Entlang der Konfliktlinie zwischen modernisierenden (NPERG, BAS) und älteren Habitusformationen (TRAK, PERG) geht es auch im religiösen Feld um den Aufstieg der »neuen« zum Schaden der »alten « gesellschaftlichen Positionen. Im modernisierenden Bürgertum überschneiden sich beide Konfliktlinien ganz besonders deutlich.

Auf welche Weise ist nun die gesellschaftliche Position der religiösen Habitusformationen bzw. Akteure in deren praktischen Logiken präsent? Zwar sind die Raumpositionen vermutlich Ausgangspunkte für die Entwicklung eines spezifischen religiösen Interesses bzw. Bedürfnisses. Aber die praktische Logik von Akteuren operiert nicht einfach linear entlang den von Soziologen beobachteten gesellschaftlichen Teilungen. Für Akteure kann eine Vielzahl spezifischer Interessen, symbolischer Differenzen etc. relevanter sein als die (noch so klar analysierten) Gesellschaftsstrukturen. Da Logik der Praxis und praktische Logik sich auf ein und dieselbe Praxis beziehen und um das Verfahren nicht sozialstrukturell zu präjudizieren, müssen nun die Operationsweisen der inkorporierten praktischen Logiken untersucht werden.

\section{Praktische Logik: Deutung, Transformation und Netzwerk}

Zur Rekonstruktion der praktischen Logiken der beiden Bewegungen werden am empirischen Material zunächst die grundlegendsten Operationen der Deutung von Krisenerfahrung untersucht. Dann werden die logischen Transformationen genauer betrachtet und schließlich wird die innere Struktur des Netzes kognitiver Operatoren entwickelt. Dieser Untersuchungsgang fokussiert kognitive Gehalte und ihre Relationierung in den Operatoren. Dazu greife ich - in Affinität zu Bourdieu - auf Methoden des französischen Strukturalismus (Greimas) zurück, binde sie aber ein in Bourdieus Theorierahmen. 


\subsection{Isotopien}

Nicht die gesellschaftlichen Krisen als solche rufen Mobilisierung hervor, sondern die Unzufriedenheit bestimmter Akteure mit ihnen. Letztere entsteht über die habitusbedingten Ursachenzuschreibungen, Bewertungen und Schlussfolgerungen, die die Akteure mit den Krisen assoziieren.

Interviewsequenzen, in denen die Informanten Probleme artikulieren, bilden den Einstieg für den ersten diskursanalytischen Schritt: die Rekonstruktion von den beiden zentralen »Isotopien «15. Dieser Arbeitsschritt erschließt kohärente Interpretations-Hinsichten für ganze Habitusformationen. In Alltagssprache: Isotopien erlauben, aus einer Vielzahl von Äußerungen das gemeinsame Grundthema der jeweiligen untersuchten Gruppe zu ermitteln und dabei die Einzelthemen festzuhalten. In der fraglichen sozialen Gruppe werden die Einzelthemen unter der Gesamthinsicht des Grundthemas gesehen, und diesem werden umgekehrt jene assoziiert. Damit enthält eine Isotopie schon Hinweise auf die semantischen - und so auch logischen - Verknüpfungen von Inhalten in der fraglichen praktischen Logik (die später untersucht werden).

Zur Rekonstruktion der Isotopien wandle ich Greimas' Semiologie im Blick auf die praktische Logik leicht ab. Ich ändere seinen »Funktionsbezug« in Erfahrungsbezug: Die entsprechenden »E-Isotopien« (Erfahrungs-Isotopien) werden in der vorliegenden Untersuchung aus Textsequenzen gewonnen, die Erfahrungen der

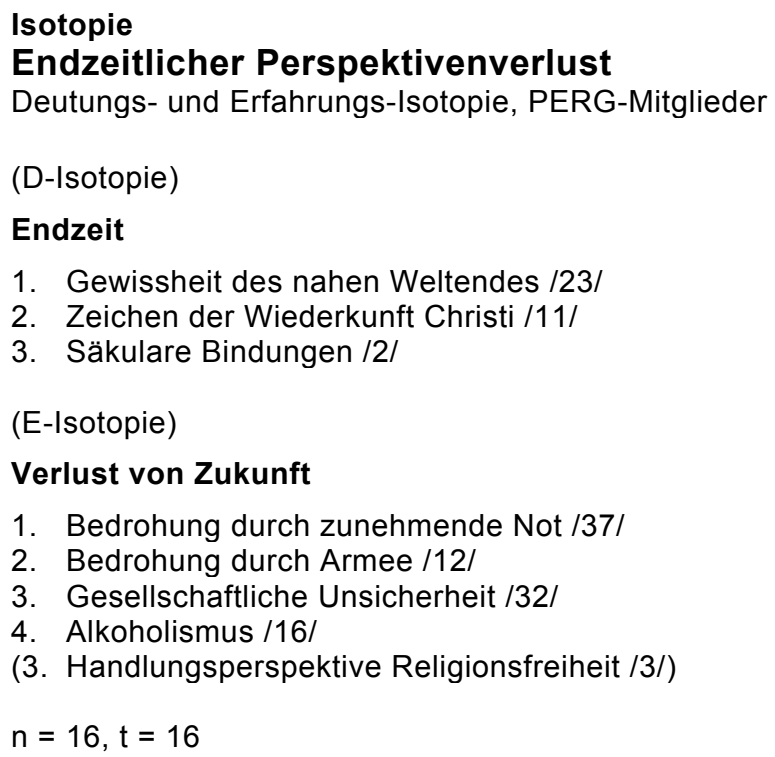

2. Zeichen der Wiederkunft Christi /11/

3. Säkulare Bindungen /2/

(E-Isotopie)

Verlust von Zukunft

1. Bedrohung durch zunehmende Not /37/

2. Bedrohung durch Armee /12/

3. Gesellschaftliche Unsicherheit /32/

4. Alkoholismus /16/

(3. Handlungsperspektive Religionsfreiheit /3/)

$\mathrm{n}=16, \mathrm{t}=16$

Abbildung 2

15 Man könnte auch »gleichthematische Reihen« sagen (A. J. Greimas, Strukturale Semantik, Braunschweig 1971). 
Akteure zum Gegenstand haben. Seinen »Aktantenbezug « transformiere ich in Deutungsbezug: Die »D-Isotopien « setzen sich aus Textsequenzen zusammen, die entsprechende Deutungen der Akteure zum Gegenstand haben. Die folgenden Ausführungen entwerfen das Modell am Beispiel von PERG/U; von NPERG werden nur die Resultate hinzugezogen.

Krisenartikulation: Verlust von Zukunft. Die Isotopie »negative Lebensbedingungen «16 (eine E-Isotopie) stellt die Wahrnehmung dar, die die PERG/U-Mitglieder von Krisen und Problemen haben: die objektiv festgemachte Unzufriedenheit mit den Lebensbedingungen. Sie verdichtet sich im Oberbegriff »Verlust von $\mathrm{Zu}$ kunft«, d. h. Verlust von Lebensmöglichkeiten und Handlungsperspektiven. Diese Wahrnehmung hat starken Anhalt in der gesellschaftlichen Entwicklung. Die Akteure verstehen den Verlust von Zukunft als Syndrom aus wirtschaftlicher Not, militärischer Gewalt und einer allgemeinen gesellschaftlichen Unsicherheit aufgrund der Zerrüttung verschiedenster sozialer Lebenszusammenhänge wie öffentliche Sicherheit, Familie, indigene Dorfstruktur und politische Institutionen; Alkoholismus wird zu einem äquivalenten Problem im Feld des persönlichen Lebens. Besonders wichtig ist die Wahrnehmung, dass »alles immer schlechter wird «, dass also keine positive soziale Laufbahnperspektive mehr besteht. Diese Wahrnehmung wird oft fast sprichwörtlich zusammengefasst: No hay para donde! - Es gibt kein Wohin!

Was sich aus wissenschaftlicher Distanz als Verlust von Zukunftsperspektiven beschreiben lässt, zeigt sich aus der Sicht der Akteure als realer Verlust von Zukunft. Die »Krise vernichtet erwartbare Zukunft «. ${ }^{17}$ Eben diese Erfahrung wird von den Mitgliedern der PERG/U mit einer solchen Intensität gemacht, dass sie sie auf die »ganze Welt« ausweiten. Ihre Perspektive wird apokalyptisch.

Ursachenzuschreibung: Endzeit. Die Mitglieder der PERG/U deuten den Verlust von Zukunftsperspektiven als Zeichen dafür, dass das Ende der Welt nahe ist. Die D-Isotopie zu den Lebensbedingungen spitzt sich auf den Oberbegriff »Endzeit « zu. Not, Gewalt, soziale Zerrüttung etc. werden darauf zurückgeführt, dass die Welt ihrem Ende in einem gesellschaftlichen und kosmischen Chaos entgegengeht. »Kriege und Hungersnöte « prägen das Geschehen, und zwar unwiderruflich, da sie als »Erfüllung des Wortes Gottes « gelten. Zugleich kündigen sie zeichenhaft die Wiederkunft Christi auf den Wolken des Himmels an. Der »Teufel« als ein möglicher Akteur (den man in zweiter Instanz auch mit dem Militär identifizieren kann) wird nur am Rande erwähnt. Und selbst wenn, wie selten, von der Bosheit der Menschen die Rede ist, wird diese nur als notwendiges Phänomen der Endzeit gesehen. Der Ton dieser Isotopie liegt somit klar auf der Unwiderruflichkeit des Geschehens und seiner quasi-kosmischen Notwendigkeit. ${ }^{18}$

16 Aus Raumgründen können hier keine detaillierten Isotopien, sondern nur Zusammenfassungen dargestellt werden (Abb. 2).

17 O. Rammstedt, Soziale Bewegung, Frankfurt 1978, 139.

18 »Die Bibel selbst sagt, wenn sich das Ende der Welt nähert, wird alles dieses geschehen. Es muss Krieg geben, (vgl. Matthäus 24,6) es muss Gewalt geben (...) denn es gibt kein Wo- 
Deutung der Erfahrung: Endzeitlicher Perspektivenverlust. Erste Deutung entsteht, wenn Beschreibung und Ursachenzuschreibung zusammengezogen werden. Die praktische Logik leistet dies sparsam und treffend, wenn etwa ein biblisches Zitat gleich als Lagebeschreibung gebraucht wird. 19

Bei der analytischen Rekonstruktion der Tiefenstrukturen können folglich Eund D-Isotopie unter eine gemeinsame »Klassem-Basis « (Greimas) zusammengefasst werden: Der Verlust von Zukunft erklärt sich aus dem Anbruch der Endzeit, so dass die Krise von den Akteuren als ein endzeitlicher Perspektivenverlust verstanden wird. Die Isotopie der Krisenerfahrung und Ursachenzuschreibung zusammen lautet also Endzeitlicher Perspektivenverlust.

Ansatz für Alternativen: Endzeitliche Errettung. In der Diskursanalyse kann man den Entwurf von Alternativen zur Krise rekonstruieren, indem man aus den semantischen Relationen, die in der Isotopie Endzeitlicher Perspektivenverlust angelegt sind, weitere Isotopien erschließt. Eine vorsichtige logische Formalisierung der semantischen Relationen erlaubt, konträre, implikative und kontradiktorische Relationen zu bestimmen sowie die Terme zueinander in Beziehung zu setzen. Aus den entsprechenden Textsequenzen lassen sich damit präzis relationierte weitere Isotopien konstruieren, die Auskunft über die von den Akteuren wahrgenommenen Deutungs- und Handlungsalternativen geben.

Bei der PERG/U kommt so die zweite wichtige Isotopie zustande, die der Endzeitlichen Errettung. Die Mitglieder der Kirche erwarten, in unmittelbarer Zukunft bei der Wiederkunft Christi aus den Leiden der Endzeit von Gott in den Himmel hinweggenommen zu werden. Diese Erwartung impliziert, dass sich die Gläubigen durch eifrigen Kirchenbesuch auf die Entrückung vorbereiten. Sie erschließen sich dadurch ein exklusiv religiöses Praxisfeld.

Aus dem Blickwinkel der Modellkonstruktion ist durch die Gegenüberstellung der beiden zentralen Isotopien faktisch schon die zentrale Tiefenstruktur bestimmt. Dabei bilden die beiden E(rfahrungs)-Isotopien und die beiden $\mathrm{D}$ (eutungs)-Isotopien je eine semantische Achse (Abb. 3). Auf der Erfahrungsebene ist dies die Kontinuität des geschichtlichen Daseins; auf der Deutungsebene die Diskontinuität des Abbruchs der Geschichte in der Endzeit. Praktisch gesagt: Die PERG/U Mitglieder sind, recht zutreffend, überzeugt, dass sie nur dann am Leben bleiben können, wenn sie sich von der Welt abwenden und in ihrer Gruppe isolieren. Dieser Grundstruktur folgend, lässt sich die für die PERG/U zentrale Transformation gesellschaftlicher Erfahrung in der religiösen praktischen Logik nachzeichnen.

hin. Also hat Christus gesagt: >Wenn ihr alle diese Dinge seht, dann deshalb, weil das Reich des Herrn nahe ist< (vgl. Markus 13,29). Das ist jetzt; heutzutage sind wir in den letzten Zeiten.« (Interview 12).

19 »>Volk gegen Volk, Sohn gegen Vater und Vater gegen Sohn.く (Matthäus 24,7) Es erfüllt sich schon.«(Interview 12). 


\subsection{Quadrat}

Die bisherige Analyse hat eine vierpolige semantische Struktur ergeben. Die Anordnung von Zeichenoppositionen reicht allerdings nicht aus. »Meaning construction $\ll 20$ und das identitätsprägende Zusammenspiel von »ends, means and environment $\ll^{21}$ bei religiösen Bewegungen sind Prozesse. Eine praxeologische Untersuchung muss also auch die Umwandlungen zwischen Erfahrung, Wahrnehmung, Urteil und Handeln in den Blick bekommen. Diese Transformationen lassen sich durch eine generative Struktur in logischen Schritten modellieren. Die Struktur bildet in einem späteren Stadium das Zentrum eines weiten Netzes praktisch-logischer Operatoren. Das Modell des »semiologischen Quadrats «22 kann in diesem Interesse adaptiert werden.

Das praxeologische Quadrat: In Anlehnung an eine mittelalterliche Vorlage organisiert dieses Modell drei grundlegende Relationen der aristotelischen Logik (Implikation, Kontrarietät und Kontradiktion) in einer Struktur logischer Übergänge: Von A über Nicht-A zu B, und von B über Nicht-B zu A; z. B. von »passiv« über »nicht-passiv« zu »aktiv«. Greimas und Rastier verwenden das >semiologische Quadrat< zur Organisation von Tiefenstrukturen des >semantischen Universums<. Zur soziologischen Verwendung ist es nötig, die Funktionslogik des Modells im Blick auf Erfahrungs- und Feldbezug zu verändern. Nicht das Zeichensystem selbst, sondern der Gebrauch von Zeichen durch die Akteure, ihr Erfahrungs- und Handlungsbezug sowie die Zeichenfunktion von Praktiken und Gütern sind von Interesse. Überträgt man das Modell aus der Begriffslogik in die Aussagenlogik, so kann man die semiotische Beschränkung aufheben. Aus dem semiologischen wird ein praxeologisches Quadrat - kompatibel mit Bourdieus Theorierahmen.

So ist es möglich, den Umwandlungsprozess ausgehend von der Krisenerfahrung (Problemartikulation) der Akteure zu beschreiben. Damit setzt die Konstruktion des Modells bei Feldbezügen an, die für die Akteure relevant sind. Alle weiteren Schritte der kognitiven und wertenden Transformationen, über die Deutung bis zum Handlungsentwurf, sind somit auf die Problemartikulation bezogen. Die Terme des Transformationsmodells (Abb. 3, Positionen A, B, $\underline{\mathrm{A}}$ und $\underline{\mathrm{B}}$ werden durch die Diskursanalyse mit Inhalten belegt. Seine logischen Relationen können ebenfalls semantisch gefüllt und so einer gehaltvollen Interpretation zugänglich gemacht werden.

Transformationsmodell: Das Modell erlaubt, die kognitive Umwandlung negativer in positive Erfahrung und in Handlungsentwürfe nachzuzeichnen, und zwar über die epistemische und die handlungsorientierte Transformation (Abb. 3) - Prozesse, die auch der Hervorbringung von Identität und Strategie zugrunde liegen.

20 R. Benford; D. Snow, »Framing processes and social movements«, in: Annual review of Sociology 26, 2000, 611-639, hier: 615.

21 A. Melucci, »Getting involved«, in: International social movement research 1, 1988, 329348, hier: $332 \mathrm{f}$.

22 A. J. Greimas; F. Rastier, »Les jeux des contraintes semiotiques«, in: A. J. Greimas, Du sens, Paris 1970, 135-155. 


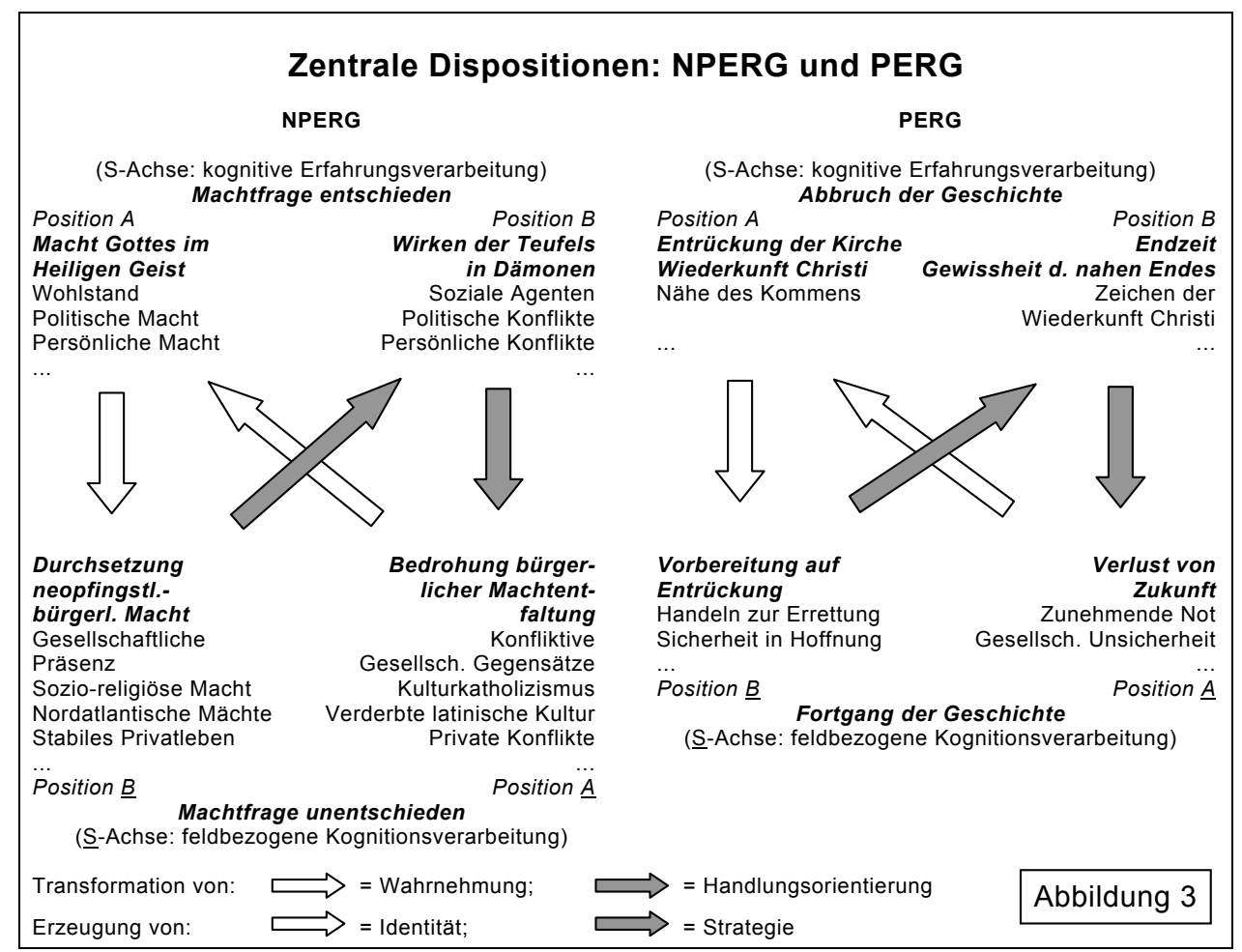

Die epistemische Transformation ( $\underline{\mathrm{A}}$--> A --> $\underline{\mathrm{B}})$ setzt an bei der Krisenerfahrung (Problemartikulation, $\underline{\mathrm{A}}$ ), stellt ihr einen Lösungsansatz (A) entgegen und bestimmt davon ausgehend die neue Stellung der Akteure (B) $)$. Die Habitusformation PERG/U geht aus von einem Verlust von Zukunft ( $\underline{\mathrm{A}})$, setzt diesem die Hoffnung auf die Entrückung der Kirche in den Himmel (A) entgegen und bestimmt von dort her ihre eigene Position als Vorbereitung auf die Entrückung ( $\underline{B})$. Diese Transformation erschließt die Stellungnahme der Bewegung gegenüber der Krisensituation; sie erzeugt Identität. Die handlungsorientierte Transformation ( $\underline{B}-->B$ B --> $\underline{A}$ ) beschreibt die Strategie, mit der von der neuen Position (B) ausgehend die alten Probleme ( $\underline{\mathrm{A}})$ gelöst werden sollen. Sie operiert mittels einer Ursachenzuschreibung zur Krise (B), welche natürlich dem Lösungsansatz (A) konnotiert ist, der der Bewegung vorschwebt (semantische Achse A -- B). ${ }^{23}$ Die Gemeinde in der Vorbereitung auf die endzeitliche Entrückung ( $\underline{B})$ führt den Verlust von Zukunft (스) auf den Anbruch der Endzeit (B) zurück. Die Strategie ist Rückzug aus der Welt. Die

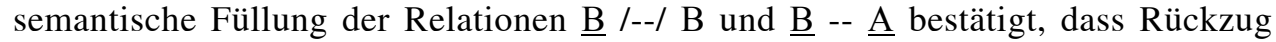
das grundlegende Handlungsschema von PERG/U ist (gegenüber Exorzismus, Austreibung der Gegner, bei NPERG). Die kognitive Verarbeitung von Erfahrung wird

23 Logische Relationen: --> lies »Implikation«, /--/ »Kontradiktion« und -- »Kontrarietät«. 
somit immer schon im Zusammenhang von vorteilhaften und nachteiligen Handlungsbedingungen, von Chancen und Begrenzungen der Akteure, modelliert. $\mathrm{Zu}$ dem stellt das Modell die Strukturierung und Wertung von Erfahrung und den Entwurf von Strategien als homologe Prozesse dar. Das entspricht der gut belegten Annahme, dass Wahrnehmungen und Handlungsentwürfe (wenn schon nicht immer vollzogene Handlungen) von wirklichen Akteuren sich meist einigermaßen kohärent zueinander verhalten. Strategien verstehen sich somit von Identitäten her, was allerdings die Möglichkeit des rationalen strategischen Kalküls nicht ausschließt. ${ }^{24}$

Durch die Modellierung empirischer Untersuchungsergebnisse in einem Transformationsmodell praktischer Logik kann somit nachgezeichnet werden, wie Erfahrungsverarbeitung über religiöse Deutung Grundstrukturen praktischer Logik (und kollektiver Identität) hervorbringt. Das Modell berücksichtigt die semantischen, logischen, strukturalen und generativen Dimensionen. In die Transformation fließt maßgeblich die gesellschaftliche Position der Akteure ein. Ein erster Eindruck davon lässt sich über einen Vergleich der zentralen Transformationen bei PERG/U und NPERG gewinnen.

Empirische Differenz: Apokalyptik versus Charismatik. Die beiden untersuchten Habitusformationen PERG/U und NPERG sind aus einem gemeinsamen Zeicheninventar und unterschiedlichen gesellschaftlichen Positionen hervorgegangen. Der Vergleich der Grundstrukturen praktischer Logik beider Bewegungen (Abb. 3; mehr zu NPERG in Kap. 4.3) zeigt Folgendes: Aus der Position gesellschaftlich marginalisierter und in eine Überlebenskrise gedrängter Akteure entsteht eine apokalyptische Identität; aus der einer modernisierenden oberen Mittelschicht mit Durchsetzungsschwierigkeiten eine charismatische. Bei PERG/U geht es um Überleben zwischen dem apokalyptischen Abbruch der Geschichte und dem Fortdauern der Leiden; bei NPERG um soziale Interessensdurchsetzung mittels der Macht des Heiligen Geistes. PERG/U setzt gegen die Existenzbedrohung eine »Zukunft im Himmel«, die »irdisches Handeln« zwar desavouiert, den Mitgliedern aber religiöse Gemeinschaften als Rückzugsräume zur Identitäts- und Existenzbehauptung durch Abgrenzung schafft. ${ }^{25}$

NPERG setzt gegen vielfältige Bedrohungen der Durchsetzungsfähigkeit ihrer Mitglieder religiöse Zueignung von Macht des Heiligen Geistes durch ekstatische Inkorporierung in einzelne Personen, inhaltlich differenziert nach Praxisfeldern. ${ }^{26}$

Unterschiedliche gesellschaftliche Positionen bringen unterschiedliche identitäre Dispositionen hervor, sogar aus demselben Zeicheninventar. Das vierpolige analytische Modell der praktischen Logik hat somit schon eine unserer Forschungsfragen beantwortet. Die Analyse der praktischen Logik selbst drängt darüber hinaus auf die Erweiterung des Modells zu einem Netzwerk.

24 ...wohl aber das rationale Tabula rasa-Individuum des methodologischen Individualismus.

25 »Ich erwarte die Wiederkunft Christi, denn es gibt kein Wohin mehr, es gibt keinen anderen Weg, denn der Herr sagt in seinem Wort: >Meine Seele wird auffliegen... Wenn du dich nicht vorbereitest, wirst du aber hier bleiben.« (Interview 33).

26 »Es ist unwichtig, wann Dein Reich kommt, aber gib uns Macht, uns zu entfalten in der Umgebung, mit der wir zu tun haben.« (Rede 107). 


\subsection{Netzwerk}

Aus der Untersuchung der inneren Relationen der Diskurse ergibt sich quasi von selbst die Erweiterung der vierpoligen Struktur zu einem Netzwerk der wahrscheinlichsten »Gedankenschritte« einer praktischen Logik. Das Modell operationalisiert die Überlegungen Bourdieus zur strukturellen Vernetzung praktischer Logik $^{27}$ für die empirische Forschung. Es zwingt der praktischen Logik dabei keine Überprägnanz auf, sondern verfolgt am empirischen Material ihre Operationen. Es erlaubt, vielfältige Transformationsschritte nachzuzeichnen. Die Analyse homologer Relationen zeigt Übertragungen von Wahrnehmungs- und Handlungsmustern zwischen verschiedenen Feldern und erlaubt die Einschätzung ihrer Relevanz für Identität, Strategie und Kapitaleinsatz. Die Logifizierung ermöglicht, Brüche, Paradoxien, leere Stellen etc. zu erkennen und aus dem Feldbezug der praktischen Logik zu erklären. Die Wirkung von Praxisfeldern, Positionen und sozialen Kämpfen auf praktische Logiken werden damit erkennbar.

Methodisch orientiert sich die Entwicklung des Netzwerks an semantischen Relationen. Durch die Nutzung der diskursiven Tiefenstrukturen kann man aus der zentralen Struktur der praktischen Operatoren weitere, untergeordnete Strukturen herausarbeiten. So verweist etwa die E-Isotopie »Vorbereitung auf die Entrückung « (Position B) bei PERG/U auf eine weitere Isotopie: Kirche. Deren Relationen bringen ihrerseits eine weitere hervor: Gottlose Welt, etc. Diese und viele weitere formen sich in der Analyse zu einem Netz von Einheiten, die untereinander formal homolog sind.

Bei der apokalyptischen Identität der PERG/U (Abb. 4) etwa wird - über die zentrale Struktur von Endzeit und Entrückung der Kirche hinaus - erkennbar, dass Gehorsam (D) gegenüber den kirchlichen Autoritäten (C) als Äquivalent zur Vorbereitung $(\underline{B})$ auf die Entrückung (A) angesehen wird. Übung in Heiligkeit (C2) gilt als Quelle von Solidarität (므) in den Gemeinden, welche wiederum äquivalent ist zur Hilfe durch Wunder (B2), durch welche Gottes Segen (A2) selbst in der Endzeit (B) noch erfahrbar ist. Entrückung (A) ist indirekt äquivalent zur Zuflucht in der Kirche (B1), weshalb man gesellschaftliche Probleme hinnehmen sollte (A1) etc. Das wichtigste Handlungsschema, der Rückzug aus der »Welt«, wird von der zentralen Struktur auf andere übertragen, etwa als Abstand von der Politik in Ablehnung menschlicher Bosheit (ㅁ3 /--/ D2), als Abgrenzung vom Weltlich-Sein der falschen Kirche (H, $\underline{H} 1)$ etc.

Der Identitätstyp von NPERG (Abb. 5) hingegen ist orientiert an Charisma und Macht. Die praktische Logik setzt an bei der Bearbeitung privater Krisen (Alkoholismus, Bulimie, Eheprobleme, A). Sie führt die Krisen auf das Handeln von Dämonen (B) zurück und schreibt dagegen den neopfingstlichen Individuen die Macht des Heiligen Geistes (A) zu, woraus die Fähigkeit zum Austreiben der Dämonen (B /--/ B) resultiert. Zugrunde liegt die Erfahrung, dass die Machtfrage in den gesellschaftlichen Beziehungen nicht entschieden ist ( $\underline{B}$-- A, $\underline{C}--\underline{D})$. Schuld daran ist der Teufel (D). ${ }^{28}$

27 P. Bourdieu, Sozialer Sinn, Frankfurt 1987, 466.

28 »Satan hält die Welt in diesem Elend und in diesem Chaos, in dem wir uns befinden.« (Interview 101/121). 
Netz der praktischen Operatoren/kognitiven Dispositionen, PERG, Mitglieder

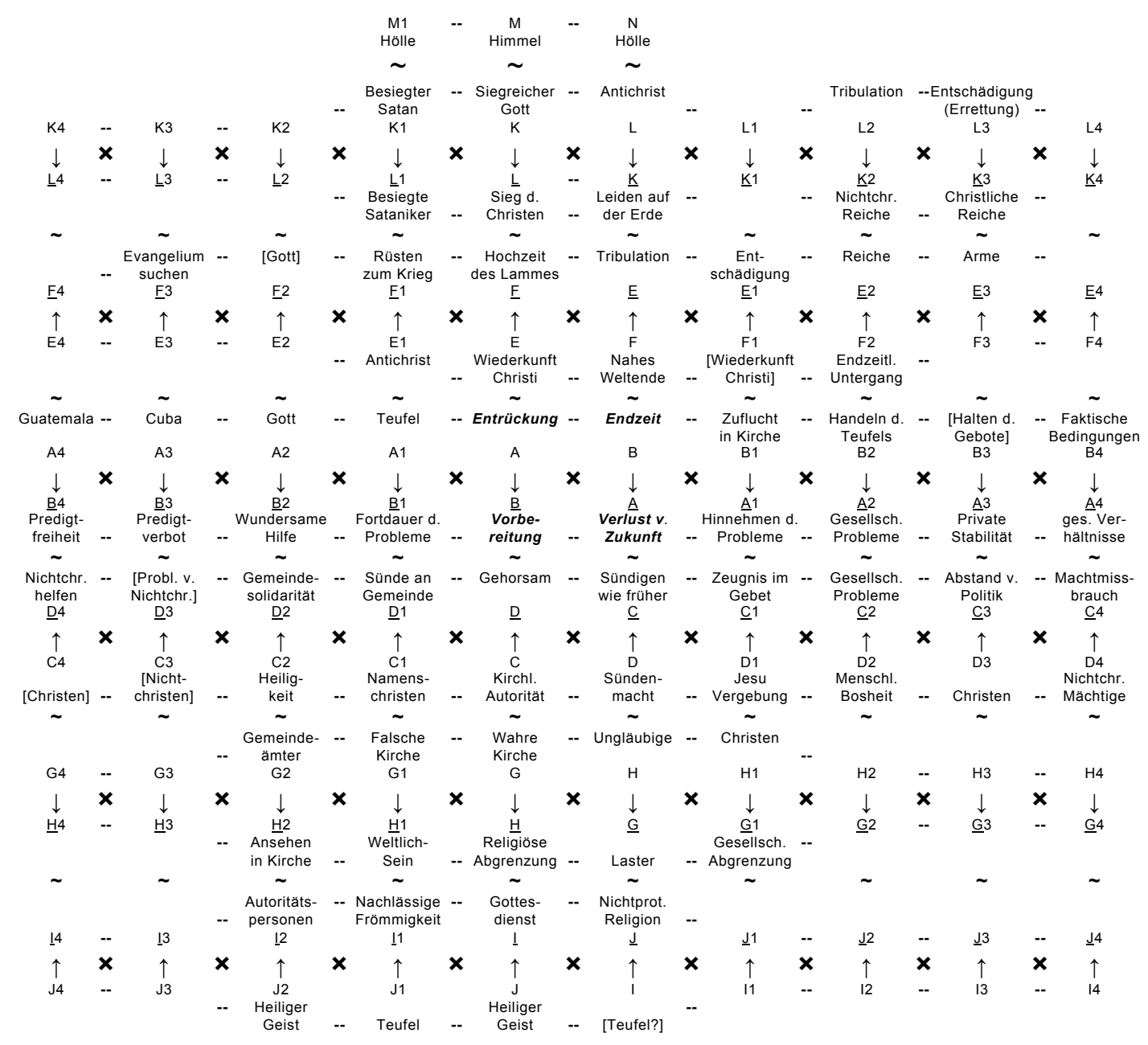

Zentrale Struktur: A, B, $\underline{A}, \underline{B}$

$\mathbf{X}=$ jeweils zwei Kontradiktionen, z. B.: A /--/ $\underline{A}$ und B /--/ $\underline{B} ; \uparrow$ und $\downarrow=$ Implikation; $--=$ Kontrarietät; $\sim=$ Äquivalenz.

A, B, C ... D D-Isotopien; $\underline{A}, \underline{B}, \underline{C} \ldots=$ E-Isotopien

Abbildung 4 


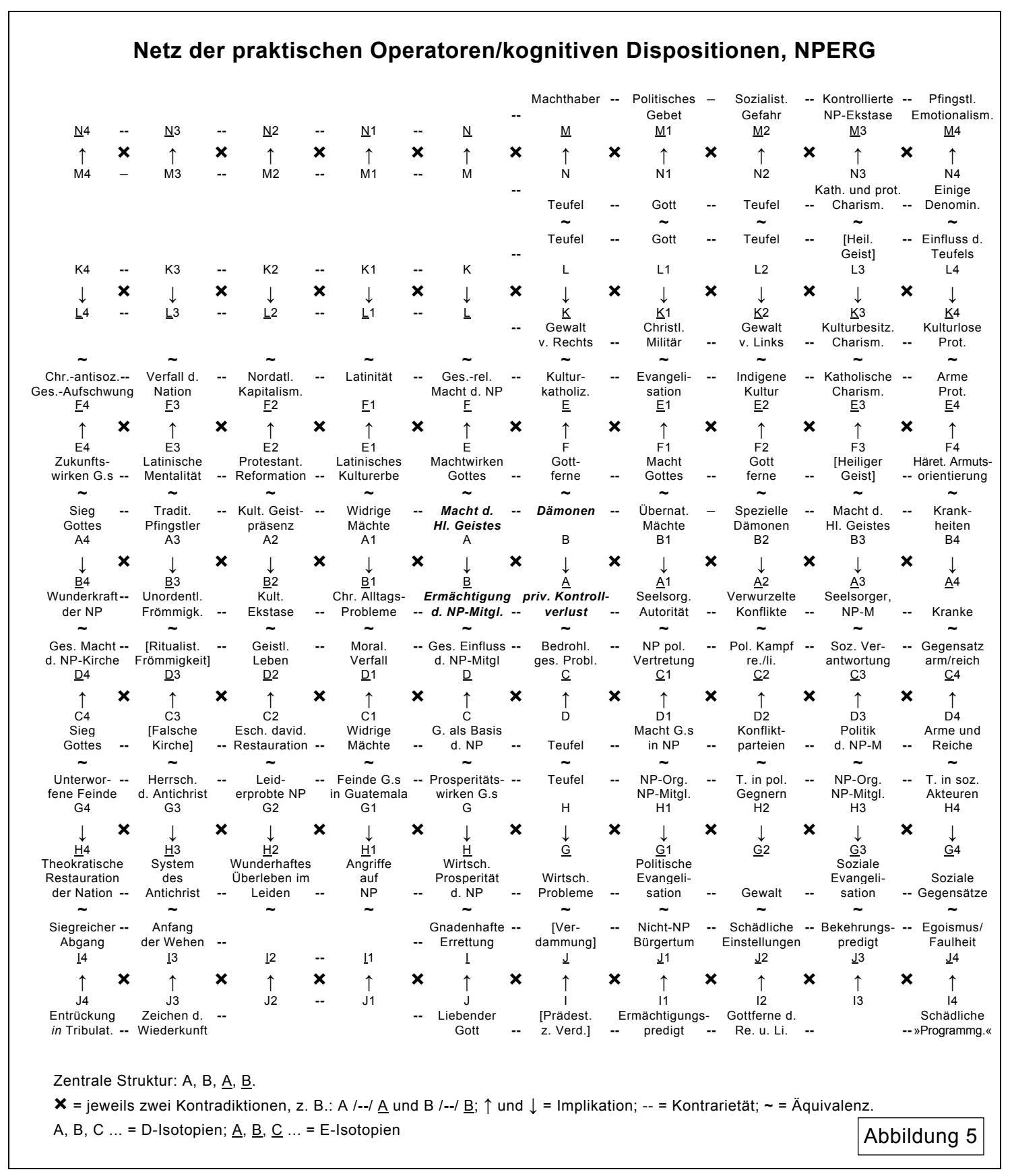


Diese unentschiedene Lage wird auf der Ebene der Deutung durch Klassifizierung und Wertung eindeutig entschieden: NPERG ist von Gott (E, F1, A4...), ihre Gegner sind vom Teufel (H2, L, L2). NPERG projektiert das Handeln ihrer Anhänger auf die Gesellschaft. Dabei bezieht sie weite Bereiche der Gesellschaft differenziert in eine religiöse Wertung ein, statt wie die PERG/U die ganze »Welt« abzulehnen. Gemäß dieser Logik greift Gott selbst in die Konflikte ein durch die Macht des Heiligen Geistes (A), durch ermächtigte NPERG-Mitglieder (B), durch ihr politisches Wirken ( $\underline{\mathrm{C}} 1)$ und ihre soziale Verantwortung ( 3$)$, durch eine protestantische Reformation (E2) und christliches Militär (K1). Aktionsziele sind wirtschaftliche Prosperität der Mitglieder ( $\underline{\mathrm{H}})$ und theokratische Restauration ( $\underline{\mathrm{H}} 4)$. Probleme und gegnerische Positionen werden satanisiert. Spezielle Dämonen (B2) bringen die Menschen psychisch gegen sich selber auf (므). Widrige Mächte (A1) schaffen Probleme (B1) und werden dem latinischen Kulturerbe (E1) zugerechnet. Sie sind aktiv in den Feinden Gottes in Guatemala (G1), die die NPERG angreifen $(\underline{\mathrm{H}} 1)$, in den politischen Gegnern (H2), welche allgemeine (G2) und militärische Gewalt von links und von rechts $(\underline{\mathrm{K}} 2, \underline{\mathrm{K}})$ hervorrufen etc. Alles in Allem werden hier sehr schnell eindeutige Zuschreibungen vorgenommen: Gott oder der Teufel.

Die beiden Netze praktischer Operatoren differenzieren die unterschiedlichen religiösen Identitäten weiter und eröffnen Möglichkeiten, die Übertragung der religiösen Dispositionen auf andere Praxisfelder besser zu verstehen. Entscheidend ist freilich, dass sie das Zusammenspiel von praktischer Logik und Logik der Praxis, von Dispositionen und Positionen, genauer zu beschreiben erlauben.

Zur Beantwortung unserer Ausgangsfragen leistet das Modell eines Netzwerks praktisch-logischer Operatoren Folgendes:

- Es erlaubt, »Landkarten« praktischer Operatoren unter Berücksichtigung der gesellschaftlichen Position der Akteure zu rekonstruieren.

- Es setzt bei der Krisenerfahrung der Akteure an und modelliert von dort ausgehend die kognitiven Transformationen, die zur Stellungnahme und zu Strategien der Akteure führen.

- Es modelliert die Übertragung von Schemata zwischen Feldern.

- Es hält den Bezug auf gesellschaftliche Erfahrung in den kognitiven Operatoren präsent.

Damit beantwortet das Modell klar unsere erste Forschungsfrage: Gesellschaftliche Positionen haben - zumindest in unserem empirischen Fall - einen starken Einfluss auf religiöse Dispositionen. Die zweite Frage - wie im Einzelnen die gesellschaftlichen Strukturen in den praktischen Operatoren präsent sind - ist damit noch nicht vollständig beantwortet. 


\section{Positionen und Dispositionen: sozialer Raum, Felder und praktische Logik}

Wo also zeigen sich genau beschreibbare Entsprechungsverhältnisse zwischen Logik der Praxis und praktischer Logik, zwischen der objektiven und der subjektiven Dimension der Praxis religiöser Akteure? Wenn man bei der Sozialstruktur einsetzt, lässt sich diese Frage nur unzureichend beantworten. Denn die praktische Logik operiert nicht gemäß der von Soziologen analysierten, sondern entlang der von den Akteuren als relevant erfahrenen - wenngleich keineswegs immer bewussten - Strukturen. Das bedeutet aber nicht, dass es keine Entsprechungen gäbe. Es besagt nur, dass Entsprechungen am besten ausgehend von der - in diesem Fall religiösen - praktischen Logik gefunden werden können.

Das religiöse Feld unterscheidet sich von anderen Praxisfeldern (z. B. Ökonomie, Politik, Recht) durch ein hohes Maß an Eigendynamik. ${ }^{29}$ In weiten Teilen profiliert es sich geradezu gegen Ökonomie und Politik. ${ }^{30}$ Das heißt aber (auch bei Weber) gerade nicht, dass es gänzlich abgelöst wäre vom Rest der Gesellschaft. Aufgrund seiner Distanz zu Politik, Ökonomie etc. bietet das religiöse Feld Akteuren vielmehr die Chance, sich religiös zu organisieren, wenn sich ihre anderweitigen Ressourcen erschöpfen. Religion (ähnlich wie ethnische Zugehörigkeit) eröffnet eine neue Perspektive auf die ökonomischen und politischen Probleme und erschließt innovative Strategien (z. B. Selbstausgrenzung oder Exorzismus) zur Bewältigung sozialer, politischer oder ökonomischer Krisen. ${ }^{31}$ Gerade der Unterschied des religiösen Feldes zu anderen Praxisfeldern ermöglicht eine kreative Anwendung religiöser Logik auf andere Felder. In diesem Sinne lässt sich präzisieren, wie die »aus der Art der Gottesvorstellungen und des > Weltbildes < folgende rationale religiöse Heilspragmatik... Folgen für die Gestaltung der praktischen Lebensführung «32 zeitigt. Religiöse praktische Logik ist zwar »gleichnishaft und symbolisch«, aber dennoch recht zuverlässig auf die gesellschaftlichen Verhältnisse bezogen. ${ }^{33}$ Doch sie bildet die Gesellschaft nicht ab. Sie steht vielmehr in einem (objektiv) strategischen Verhältnis zur Gesellschaft und arbeitet allenfalls mit Homologien zur Logik nicht-religiöser Praxis. Solche Homologien sind heuristisch wichtige Einstiegspunkte für die Analyse des Verhältnisses von Dispositionen und Positionen von Akteuren.

Im Folgenden werde ich das objektiv strategische Verhältnis zwischen Religion und Gesellschaft bei NPERG (3.1) und bei PERG/U (3.2) untersuchen. Dabei

29 Vgl. M. Weber, Gesammelte Aufsätze..., 240 f., 544 ff.; P. Bourdieu, Das religiöse Feld..., $11 \mathrm{ff} ., 53$.

30 M. Weber, Gesammelte Aufsätze..., $541 \mathrm{ff}$.

31 Besonders große Distanz ermöglicht besonders radikale Lösungen bei hoher Integration durch Selbst-Verabsolutierung (H. Schäfer, »Religious fundamentalism«, in: J. de Santa Ana (Hg.), Religions today, Geneva 2005). Sehr hoch integrierte Bewegungen (Fundamentalismen) bezahlen daher ihre punktuelle Durchschlagskraft mit einem Mangel an flexibler Reaktionsfähigkeit, während liberale Bewegungen (Ökologie, Globalisierungsgegner, Ökumene) bei geringerer Integration flexibler sind.

32 M. Weber, Gesammelte Aufsätze..., 259.

33 E. Durkheim, Die elementaren Formen des religiösen Lebens, Frankfurt 1981, 309. 
werde ich folgende Aspekte besonders beachten: Strukturvariablen des sozialen Raumes wie Kapitalvolumina und Konfliktlinien sowie Laufbahnperspektiven, Modernisierungsdynamik, funktionale Differenzierung, Habitusformen und Interessen der Akteure. Aus den Ergebnissen ziehe ich dann Schlussfolgerungen über die relative Unabhängigkeit des religiösen Feldes (3.3).

\subsection{NPERG: Weltbeherrschung}

Kapitalvolumen: Wir haben die religiösen Habitusformationen anhand von demographischen Kriterien den Positionen des gesellschaftlichen Raumes zugeordnet und somit gemäß Klassenlage verortet. Die Stichprobe der NPERG gehört zur Position der modernisierenden Mittel- und Oberschicht: technische Intelligenz, Freiberufler, Manager, Militäroffiziere etc.

Die praktische Logik der NPERG setzt an bei Erfahrungen des Kontrollverlustes im Privatleben (Abb. 3, A). Über die Zueignung der Macht des Heiligen Geistes $(\mathrm{A})$ zu den gläubigen Individuen $(\underline{B})$ und die Deutung des Kontrollverlustes als Bedrohung durch Dämonen (B) wird Exorzismus B /--/ B) als zentrales Handlungsschema gegen den Kontrollverlust eingeübt und auf andere Praxisfelder übertragen. Diese Logik geht von einer Situation aus, in der noch Kapital und Verwertungsmöglichkeiten vorhanden sind, aber die Verfügungsgewalt darüber strittig ist (Achse $\underline{B}--\underline{A})$.

Dieser Ansatz entspricht in hohem Maße der gesellschaftlichen Lage der modernisierenden oberen Mittelschicht Guatemalas. Die Verschlechterung der terms of trade, Guerilla und Paramilitärs sowie Korruption blockieren den Aufstieg dieser Position seit Beginn der achtziger Jahre. Ihre relativ gute Kapitalausstattung und begünstigte Stellung im Modernisierungsprozess erlauben ihr gleichwohl eine wirksame Einflussnahme auf die gesellschaftlichen Prozesse.

Die religiöse Umwandlung dieser Lage antwortet auf die Erfahrung eines Kontrollverlustes durch Instabilität und Bedrohung ( religiösen Akteure durch die Macht (das »Kapital«) des Heiligen Geistes (A) und interpretiert den Kontrollverlust als Bedrohung durch einen absoluten Feind. Auf dieser Grundlage (B) kann eine neue Strategie entworfen werden. Dazu wird die Gesamtlage über den Begriff des Konflikts definiert und zwar durch die religiöse Metapher des Exorzismus ( $\mathrm{B} /--/$ B). Mit dieser Umwandlung ist zugleich die Machtfrage symbolisch (A -- B) geklärt: Die Strategie kann also mit Siegesgewissheit und Aggressivität verfolgt werden.

Strukturell entspricht diese religiöse Logik den herkömmlichen Problemlösungsstrategien in gesellschaftlichen Positionen mit hinreichender Kapitalausstattung: Man beobachtet ein Defizit, investiert freies Kapital, erwirtschaftet ein Superavit und bekämpft die Ursachen des Defizits. Sie dramatisiert allerdings die Krisenerfahrung im modernisierenden Bürgertum durch das Exorzismus-Schema. Die Akteure interpretieren Wirtschaftskrise und Bürgerkrieg somit kompromisslos als Angriffe externer Mächte und wandeln die (passive) Bedrohungserfahrung in eine exorzistische Strategie der Exklusion um. 
Dadurch gelingt es ihnen auch, ihre Laufbahnperspektiven anders wahrzunehmen. Religiös gesehen ist ihr Aufstieg dann nicht mehr blockiert. Das göttliche Machtwirken eröffnet vielmehr neue Chancen: wirtschaftliche Prosperität (Abb. 5, $\underline{\mathrm{H}})$ und theokratische Restauration ( 4$)$.

Angesichts der relativ guten Ausstattung der Akteure mit politischem, ökonomischem und sozialem Kapital leistet die religiöse Logik gegenüber der Krise eine Stabilisierung der Akteure und eine Verbesserung der Verwertbarkeit ihres Kapitals durch die Entwicklung von Machtstrategien. Die praktische Logik der NPERG ist objektiv angepasst an ihre Position im sozialen Raum, deren objektiven Machtchancen und Produktionsweisen. Sie ist - dazu ganz entsprechend und im Gegensatz zur PERG/U - sehr weitgehend auch subjektiv strategisch. Das zeigt sich auch im Umgang mit den gesellschaftlichen Konflikten.

Konflikte: Das Raummodell lässt zwei für das modernisierende Bürgertum relevante Konflikte erkennen: den sozio-ökonomischen Konflikt mit dem rebellierenden (Stadt- und Land-) Proletariat (Abb. 1, Konflikt A) sowie den Modernisierungskonflikt mit der alten Oligarchie (Konflikt B 1).

Das Exorzismusschema - eine religiöse Transformationsgestalt gesellschaftlicher Aggression - antwortet in offensiver Weise auf beide Konflikte. Es passt die Akteure an die objektiven Handlungsbedingungen an und verleiht ihrem Kapitaleinsatz auf verschiedenen Feldern eine offensiv strategische Note, u. a. durch die Satanisierung gesellschaftlicher Gegner (Abb. 5, H2). Satanisierung wird auf beide Konflikte angewandt ( $\mathrm{N}->\underline{\mathrm{M}}$ und $\mathrm{N} 2$--> $\underline{\mathrm{M}} 2)$ und verstärkt so die politische und militärische Entschlossenheit. Aber sie verhindert nicht per se differenzierte Strategien.

Nimmt man beide Konflikte zusammen, so ergibt sich für das modernisierende Bürgertum eine klassische Zwischenstellung. Damit verbinden sich Chancen der Kooptation und Legitimation. Die religiöse Logik greift diese objektive Zwischenstellung auf, indem sie ternäre Strukturen bildet. In solchen Dreierstrukturen werden NPERG bzw. interessenidentische Akteure als Alternative zu jeweils zwei Konfliktparteien positioniert: NPERG als Agent der Evangelisation (E1) gegenüber der indigenen Kultur (E2) und dem Kulturkatholizismus (E); christliches Mi-

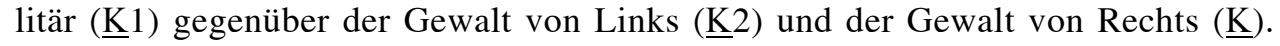
Insbesondere indem NPERG soziale Verantwortung ( $\underline{C} 3$ ) als ein Resultat ihrer politischen Intervention (D3) propagiert, ${ }^{34}$ positioniert sie sich als Alternative zu den Armen und zu den Reichen (D4) sowie zu den politischen Konfliktparteien schlechthin (D2): eine Alternative, die sowohl dem politischen Kampf zwischen Rechts und Links (므) als auch dem Gegensatz zwischen Arm und Reich (드) ein Ende bereiten kann. Die Betonung der sozialen Frage - und die Dreierstruktur generell - zeitigt Kooptations- und Legitimationseffekte: Vor der Oligarchie kooptiert NPERG die unteren Klassen und vor den unteren Klassen legitimiert sie sich durch Interessenvertretung gegenüber der Oligarchie. NPERG konstituiert sich, und damit das modernisierende Bürgertum insgesamt (»Nicht-NPERG-Bürgertum«, $\underline{\mathrm{J}} 1$ ), als politischen dritten Weg.

34 ...direkt oder durch politische Parteien wie die MAS des NPERG-Führers Jorge Serrano. 
Modernisierung: Orientiert man sich an fortschreitender Rationalisierung und Modernisierung als einem sozialstrukturellen Faktor, so ist das modernisierende Bürgertum in der guatemaltekischen Gesellschaft die fortschrittlichste Position. Entsprechende Praktiken und Diskurse sind in der religiösen Logik der NPERG von Bedeutung. So z. B. treibt der nordatlantische Kapitalismus (Abb. 5, F2) latinische Mentalität (E3) und Kulturerbe (E1) aus und wird als Frucht protestantischer Reformation (E2) begriffen. Korruption und schlechte Verwaltung gelten als schwerwiegende Fehler der Machthaber (außerhalb der Darstellung des Netzwerks); Faulheit (J4), Rückständigkeit und falscher Glaube als wichtige Gründe für Armut (F4). Der Zusammenhang von Gnade (I) und Prosperität ( $\underline{\mathrm{H}})$ ist über Prädestinationsglaube vermittelt und erinnert unmittelbar an die bekannte Affinität zwischen Calvinismus und dem >Geist des Kapitalismus< (Weber). In ihrer Ablehnung des »latinischen Klientelismus « und dem Eintreten für rationale Verwaltung und kalkulierbare Bedingungen der Kapitalverwertung sind NPERG-Mitglieder entsprechend zu ihrer objektiven gesellschaftlichen Position - die guatemaltekische Form modernen >Berufsmenschentums $<$ (Weber).

Dem entspricht auch der Ansatz der religiösen Logik beim Individuum. Im individuellen privaten Alltag wird das Exorzismus-Schema eingeübt, etwa durch Austreibung eines »Alkoholismus-Dämons«. Dann wird es auf andere gesellschaftliche Bereiche übertragen. Damit wird der für neoliberale Praxis typische Besitzindividualismus religiös rezipiert und für Positionskämpfe zugerichtet.

Differenzierung: Im Netz der religiösen Operatoren der NPERG fällt auf, dass Bezug auf sehr viele Felder gesellschaftlicher Praxis genommen wird. Das entspricht der Tatsache, dass der gesellschaftliche Gestaltungsanspruch des modernisierenden Bürgertums sich auf die verschiedensten Lebensbereiche erstreckt und dort mit diversen Rollenerwartungen konfrontiert ist. Die religiöse Logik akzeptiert allerdings nur sehr begrenzt die mit funktionaler Differenzierung einhergehende Verselbständigung der Lebensbereiche. Vielmehr soll das gesamte Leben »dem Heiligen Geist unterworfen « werden.

Entsprechend der konfliktorientierten Betrachtung der Krise wird das Exorzismusschema auf die verschiedensten Felder übertragen. Es bringt sie unter die Perspektive der differenziellen Machtchancen der NPERG-Akteure. Das politische Handeln von NPERG (Abb. 5, $\underline{\text { }} 1$ ) bekämpft bedrohliche gesellschaftliche Probleme (C) durch die Austreibung des Teufels ( $\underline{\mathrm{C}} 1 /$ /--/ D). Der nordatlantische Kapitalismus (F2) treibt die latinische Mentalität (E3) aus, um gegen den Verfall der Nation (F3) anzugehen. Oder das christliche Militär treibt der Gewalt von Links (K2) den Teufel (L2) aus, und zwar durchaus auch mit Napalm. Die Übertragungsmöglichkeiten des Exorzismusschemas sind nahezu universell. Es homogenisiert funktional differenzierte Bereiche unter einem konfliktorientierten Wahrnehmungs- und Handlungsschema und fördert eine »penetrante Christianisierung des ganzen Daseins « 35 .

Die praktische Logik von NPERG richtet sich also an der positionsspezifischen gesellschaftlichen Differenzierung aus, sucht diese aber soweit zu aufzuheben, wie

35 M. Weber, Gesammelte Aufsätze..., 124. 
es für eine homogene Machtstrategie nötig ist. Sie prägt dadurch einen gesellschaftlich relevanten religiösen Habitus, der die Vorgaben im Klassenhabitus radikalisiert.

Klassenhabitus: NPERG unterstreicht in ihrer religiösen Praxis stark den gesellschaftlichen Status ihrer Trägergruppen, z. B. durch exklusive Lokalisierung der Kirchengebäude, livrierte Diener am Eingang, Prosperitätsdiskurse etc., kurz: durch Strategien der Distinktion und der Prätention ${ }^{36}$.

Distinktion richtet sich gegen die »von unten«. Die religiöse Logik erklärt Armut aus einem Einfluss des Teufels (Abb. 5, L4), einer häretischen Orientierung (F4) oder Programmierung (I4) auf Armut oder schlicht durch Faulheit (J4). Damit belegt sie eine bekannte Vulgärform der Rechtfertigung sozialer Ungleichheit lediglich mit religiösen Gehalten - das »Weltbewusstsein « einer »verkehrten Welt«37.

Der positionsspezifische Habitus politischer Prätention de-legitimiert die traditionellen Machthaber durch den Vorwurf der Verantwortungslosigkeit und legitimiert die eigene Machtprätention durch die Behauptung, das Gemeinwohl zu vertreten. Nicht anders verfährt NPERG mit ihren religiösen Strategien sozialer Zwischenstellung (s. o.).

NPERG reproduziert somit den gängigen »Makro-Habitus« ihrer gesellschaftlichen Position im religiösen Feld, und zwar nicht als Abbild ${ }^{38}$, sondern als praktische Logik unter Einbeziehung der Interessen von Akteuren.

Interessen: Religiöse Bewegungen haben religiöse, aber auch andere Interessen. ${ }^{39}$ Hier sind zunächst die nicht-religiösen von Belang.

Grundlegend für NPERG ist das Interesse, die gesellschaftliche Krise durch eine Machtstrategie zu meistern. Deren Ziele sind Prosperität (Abb. 5, $\underline{\mathrm{H}}$ ), politische Macht ( $\underline{\mathrm{C}} 1)$ und - langfristig, aber ernsthaft - Systemwechsel zur Theokratie (브). Darin konvergieren religiöse und gesellschaftliche Interessen. >Wovon und wozu also wird Erlösung gesucht< (Weber)? Vom Kontrollverlust und zur Machtausübung. ${ }^{40}$

Das religiöse Interesse der NPERG richtet sich letztlich auf die Überwindung einer zugleich individuellen und gesellschaftlichen Krise durch Strategien der Körper- und der Weltbeherrschung, gepaart mit einer »Theodizee des Glücks « ${ }^{41}$.

Die religiösen Dispositionen der NPERG entsprechen somit in vielen Details der allgemeinen Logik gesellschaftlicher Praxis in der sozialen Position dieser Akteure. Aber sie reproduzieren die Position nicht einfach. Ihre objektive Angepasst-

36 P. Bourdieu, Die feinen Unterschiede..., $405 \mathrm{ff}$.

37 K. Marx, »Zur Kritik der Hegelschen Rechtsphilosophie. Einleitung «, in: $M E W$, Bd. 1, Berlin 1976, 378-391, hier: 378.

38 E. Durkheim, Die elementaren Formen..., 563.

39 M. Weber, Gesammelte Aufsätze..., 240 f., 252.

40 »Herr des Himmels und der Erde, gib uns Macht auf der Erde. Denn die Macht im Himmel wird nicht so bewundernswert sein wie (die Macht) hier, Amen!« (Rede 109).

41 M. Weber, Gesammelte Aufsätze..., 242. 
heit dient zur Grundlage dafür, dass NPERG im religiösen Feld positionsspezifische Ansätze der Prätention und Distinktion in eine radikale, subjektiv bewusste, religiös-gesellschaftliche Machtstrategie umwandelt.

\subsection{PERG/U: Weltflucht}

Ein kurzer Blick auf PERG/U zeigt, dass auch hier eine große Anzahl von Entsprechungen zwischen der gesellschaftlichen und der religiösen Praxis existiert.

Kapitalvolumen: Die untersuchte Stichprobe der PERG/U gehört zum unteren Bereich der mit »PERG« bezeichneten Feldposition (Abb. 1): Gelegenheitsarbeiter und Kleinbauern.

Die praktische Logik interpretiert gesellschaftliche Krisenerfahrung so, dass es keine Zukunft in der Welt mehr gibt (Abb. 3, A). Die Zukunft wird außerweltlich konstruiert: die Entrückung der Kirche in den Himmel (A) und die Vorbereitung darauf $(\underline{B})$. Im Blick auf die unabwendbare Verschlechterung der Welt in der Endzeit (B) kann man nicht handeln. Man kann sich nur abgrenzen (B /--/ B).

Diese Logik entspricht der gesellschaftlichen Position der PERG/U-Mitglieder: sehr geringes Gesamtkapitalvolumen und veraltetes Wissen. Hier erzeugen Wirtschaftskrise und militärische Repression eine weitgehende Lähmung gesellschaftlicher Aktivitäten sowie objektiv absteigende Laufbahnen. Die Kapitalausstattung ist so schlecht und die Repression so hart, dass kaum noch gesellschaftliche Handlungschancen offen stehen.

Die religiöse Umwandlung dieser Lage bedient sich des Handlungsschemas der Selbstausgrenzung. Dieses wird auf verschiedene Praxisfelder übertragen. Es sorgt unter anderem für heilsamen Abstand von der Politik (Abb. 4, C3), die für kapitalschwache Ausgegrenzte in Guatemala ohnehin nur Quelle von Gefahren ist. Das ist die Grundlage dafür, dass die Akteure unter den faktischen Bedingungen (B4) vermittels der Selbstausgrenzung aus riskanten Handlungszusammenhängen private Stabilität ( $\underline{\mathrm{A}} 3)$ herstellen können. Darüber hinaus wird die religiöse Gemeinde über die Selbstausgrenzung als wahre Kirche (G) zu einem Handlungszusammenhang gemacht, der durch Heiligkeit (C2) und Solidarität (ㅁ) gekennzeichnet ist, d. h. neue Möglichkeiten des Überlebens eröffnet.

Auch bei PERG/U wird die Laufbahnperspektive neu interpretiert. Die Tatsache einer zunehmenden Verschlechterung wird als Zeichen der Endzeit (B) aufgefasst. Sie bestätigt somit die Richtigkeit der Selbstausgrenzung.

Die religiöse Logik entspricht sehr genau den Chancen und Begrenzungen der gesellschaftlichen Position. Angesichts politischer Repression und wirtschaftlicher Not wird diese Identität aus Abgrenzung und Weltflucht zu einer Ressource für das Überleben, für Gruppensolidarität und den Erwerb von Anerkennung. Sie ist objektiv strategisch - nicht subjektiv, denn die Strategie darf als solche gerade nicht bewusst werden. So gelingt es der religiösen Logik (sozusagen hinter dem Rücken der Akteure), ihnen $\gg$ zu helfen zu leben $\aleph^{42}$.

42 E. Durkheim, Die elementaren Formen..., 558. 
Konflikte: Die apokalyptische Selbstausgrenzung ist für PERG/U auch das adäquate Verhalten angesichts des zentralen gesellschaftlichen Konflikts zwischen kapitalstarken und kapitalschwachen Akteuren (Abb. 1, Konflikt A). Der Konflikt kommt zwar als Differenz zwischen Armen (Abb. 4, E3) und Reichen (E2) im Netz der kognitiven Operatoren vor, wird allerdings ambivalent bewertet (F2, $\underline{\mathrm{K}} 2$, $\underline{\mathrm{K}} 3)$. Abstand von Politik ( $\underline{\mathrm{C}} 3)$ jedenfalls ist äquivalent zu privater Stabilität ( $\underline{\mathrm{A}} 3)$. Damit entspricht PERG/U - in religiöser Logik - einer verbreiteten Haltung in ihrer gesellschaftlichen Position, in der wirtschaftliche Überausbeutung und militärische Unterdrückung insbesondere gegen Ende des Guerillakrieges jeden Widerstand zwecklos machten. Der enge Zusammenhang zwischen gesellschaftlicher Lage und religiöser praktischer Logik zeigt sich besonders deutlich an der weiteren Entwicklung: 1993 zeigte die erneute Untersuchung eines Teils der PERG/UStichprobe, dass im Vergleich zu 1986 der Demokratisierungsprozess die religiöse Logik in Bewegung gebracht hatte. Die apokalyptische Ausgrenzung stand nicht mehr im Zentrum des Netzes der Operatoren. Der Schwerpunkt hatte sich vielmehr auf ein asketisches Ethos der (sozialen) Heiligung (C2) und der Solidarität (2) verschoben.

Der Modernisierungskonflikt (Abb. 1, Konflikt B2) zwischen traditioneller Unterschicht (Parzellenbauern, Tagelöhner, Kleinstgewerbe) und »modernisiertem « Land- und Stadtproletariat spielt für PERG/U eine wesentlich geringere Rolle. Er wird unmittelbar religiös transformiert in den Unterschied zwischen wahrer (Abb. 4, G) und falscher (G1) Kirche: Letztere (PRG und BAS) grenzt sich nicht klar genug von der Politik ab.

Modernisierung: In der traditionellen Unterschicht ist es schwer, aktuelles, verwertbares Wissen durch Bildung zu erwerben; und zudem ist diese Bevölkerungsgruppe nicht in modernisierte Produktion (industrielle Landwirtschaft oder Fertigung) eingebunden. Die weltflüchtige apokalyptische Askese der PERG/U lehnt entsprechend die Modernisierung nicht nur ab; sie sieht in ihr ein Zeichen des nahen Weltendes. Sie lehnt Wandel als solchen ab, weil er per definitionem religiosam nur Wandel zum Schlechteren sein kann.

Der traditionellen Orientierung insbesondere der ländlichen Unterschicht entspricht ein hoher Stellenwert des Kollektivs (gegenüber dem Individualismus der modernisierten Mittelschicht). Das zeigt sich in den religiösen Operatoren der PERG/U, deren >Erlösungsbedürfnis< (Weber) kollektiv vermittelt ist: Nicht der Einzelne wird in den Himmel aufgenommen (Abb. 4, A), sondern die wahre Kirche als Kollektiv ( $\underline{B}, \mathrm{G})$.

Differenzierung: Insbesondere die ländliche Unterschicht Guatemalas ist funktional noch relativ gering differenziert. Dem entspricht, dass das Netz der kognitiven Operatoren der PERG/U eine nur geringe Differenzierung nach gesellschaftlichen Praxisfeldern zeigt. Es enthält überwiegend religiöse Operatoren, bezogen auf die außerweltliche Erlösung, Abgrenzung von der »Welt« und gemeindliches Zusammenleben. Nur gelegentlich werden religiöse Handlungsweisen auf andere gesellschaftliche Praxisfelder übertragen: etwa der Gehorsam gegenüber kirchlicher Autorität (ㅁ) auf das politische Feld als Gehorsam gegenüber den Autoritäten 
(Position außerhalb der Darstellung). Die Tatsache, dass das Überleben in der Krise für PERG/U vor allem durch Abgrenzung, Anpassung und innergemeindliche Solidarität gesichert wird, führt dazu, dass selbst Ansätze gesellschaftlicher Differenzierung von der religiösen Logik nicht aufgenommen, sondern unter der strikten Kirche/Welt-Dichotomie eingeebnet werden.

Klassenhabitus: Konformität, das Sich-Einfügen ins Notwendige ${ }^{43}$, bestimmt sichtlich auch die religiösen Dispositionen der PERG/U. Allerdings werten die religiösen Operatoren die gesellschaftliche Logik um. Für ein »weltliches« junges Mädchen ist der Verzicht auf Schmuck ein Resultat der Armut, ist Schande und Frustration. Für ein pfingstliches junges Mädchen ist dieser Verzicht Ausdruck der Heiligkeit (Abb. 4, C2) und Quelle für Prestige in der Gemeinde (브). Die weltflüchtige Askese produziert ihre eigenen Werte in Umkehrung dessen, was ohnehin nicht erreichbar ist. Das Ethos der Askese wird um eine eigene, »negative « Ästhetik bereichert - eine besondere Form des Ausdrucks von Elend und der »Protestation « dagegen. ${ }^{4}$

Interessen: Das >diesseitige< Interesse der PERG/U ist das Überleben unter hoffnungslosen Umständen. Das wird erreicht durch apokalyptische Selbstausgrenzung, welche die innergemeindliche Solidarität als neuen Raum für soziales Handeln konstituiert.

Die Abgrenzungsstrategie der PERG/U in einer völlig hoffnungslosen Lage ist eine apokalyptische $»$ Theodizee des Leidens «45, eine Weltflucht, die (objektiv) auf das Überleben in der Welt zielt.

Auch die religiösen Dispositionen der PERG/U entsprechen recht detailliert ihrer gesellschaftlichen Position. Doch auch hier erweist sich die Logik religiöser Praxis keinesfalls als einfaches Abbild der Gesellschaft. Sie ist vielmehr ein (objektives) soziales Strategem der Akteure. Nur die religiöse Konstruktion eines virtuell gesellschaftsfreien Praxisfeldes (der religiösen Gemeinde) ermöglicht physisches Überleben bei gleichzeitiger religiöser Rekonstruktion menschlicher Würde. Hier wird die »Kette« nur symbolisch abgeworfen, weil sonst die »lebendige Blume« nicht einmal denkbar wäre. ${ }^{46}$

\subsection{Zur Eigendynamik des religiösen Feldes}

Die Fragestellung dieses Aufsatzes zielt nicht auf die Eigendynamik des religiösen Feldes, sondern auf das Verhältnis zwischen gesellschaftlichen Positionen und religiösen Dispositionen. Nur in diesen Grenzen kann ich hier kurz auf die Eigendynamik des Feldes eingehen.

43 P. Bourdieu, Die feinen Unterschiede..., $585 \mathrm{ff}$.

44 K. Marx, »Zur Kritik der Hegelschen Rechtsphilosophie...«, 378.

45 M. Weber, Gesammelte Aufsätze..., 244.

46 K. Marx, »Zur Kritik der Hegelschen Rechtsphilosophie...«, 379. 
Zunächst organisieren sich die Spannungen des religiösen Feldes entlang unterschiedlicher Akteurstypen ${ }^{47}$, die als Positionen des Feldes im Kampf um dessen Monopol begriffen werden können. ${ }^{48}$ Allerdings hat ihre soziale Position im konkreten Fall (also nicht in der idealtypischen Konstruktion) durchaus Einfluss auf die religiösen Strategien und Handlungschancen auch dieser Akteurstypen. ${ }^{49}$

Sodann spricht im Blick auf Glaubensüberzeugungen und habitualisierte Praktiken einiges dafür, dass »der einmal geprägte Typus einer Religion seinen Einfluss ziemlich weitgehend auch auf die Lebensführung sehr heterogener Schichten auszuüben $\ll 50$ pflegt. Gleichwohl relativieren unsere empirischen Befunde aus der Pfingstbewegung dieses Theorem in gewissem Grade. Wir haben ja gesehen, dass sich aus dem symbolischen Inventar der Pfingstbewegung in einer sozial stark polarisierten Gesellschaft zwei sehr unterschiedliche Typen von Religiosität entwickelten: eine charismatische der Welteroberung und eine apokalyptische der Weltflucht. ${ }^{51}$ Der »einmal geprägte Typus Pfingstbewegung « erfährt also eine innere Differenzierung, die sich an der sozialen Position der jeweiligen Gläubigen orientiert (ähnlich jener von monopolistischen, priesterlichen Akteuren im religiösen Feld). Diese erschöpft sich nicht darin, dass ein und dasselbe Symbol gegensätzliche Bedeutung annehmen kann, wenn es auf gegensätzliche gesellschaftliche Erfahrungen bezogen ist. ${ }^{52}$ Sie betrifft die Organisation der Operatoren praktischer Logik und damit die gesamte religiöse Praxis: Die Pfingstbewegung arbeitet traditionell mit einem bestimmten religiösen Symbolinventar für die kognitive Organisation von Raum- und Zeiterfahrung; vor allem: Heiliger Geist, millenaristische Geschichtskonzeption und persönliche Heiligkeit. Die gesellschaftlichen Interessen der religiösen Akteure aber entscheiden - jedenfalls nach unserem Befund über die Ausdifferenzierung und die Kombinationen dieses Inventars. Ist der Geist an Ämterhierarchie gebunden oder ist er eine freie Kraftwirkung gegen feindliche Agenten (Dämonen)? Wird die Zeiterfahrung post- oder prämillenaristisch organisiert? Wird Heiligkeit an Kraftwirkungen oder Askese gemessen? Kurz: Werden der gesellschaftliche Machtraum und die geschichtliche Zeit als Herausforderung zur Gestaltung oder als Anlass zum Selbstausschluss aufgefasst? Dies wird nicht durch das symbolische Inventar oder durch eingespielte religiöse Praktiken als solche entschieden, sondern nur durch deren Zusammenspiel mit den nicht-religiösen Handlungsbedingungen der religiösen Akteure.

Es sind die Operatoren der praktischen religiösen Logik, welche Symbolinventar und weitere gesellschaftliche Handlungsbedingungen miteinander vermitteln. Sie bringen in dieser Vermittlung das religiöse Inventar gerade in der Form zur Geltung, in der es unter den Bedingungen seines Gebrauchs die größtmögliche

47 M. Weber, Wirtschaft und Gesellschaft, Tübingen 1985, 259, 268, 275.

48 P. Bourdieu, Das religiöse Feld..., 11.

49 Entsprechende Beobachtungen zu Guatemala habe ich aus Raumgründen gestrichen. Siehe generell aber P. Bourdieu, Das religiöse Feld..., 77.

50 M. Weber, Gesammelte Aufsätze..., 241.

51 Dies lässt sich übrigens auch an der historischen Entstehung von Pfingst- und Neopfingstbewegung in den USA zeigen (H. Schäfer, Protestantismus in Zentralamerika..., 58 ff.).

52 P. Bourdieu, Das religiöse Feld..., 76. 
religiöse Dynamik entfaltet: als Apokalyptik bei den Ausgegrenzten und als Charismatik bei den blockierten Aufsteigern. Die religiösen Operatoren sind gesellschaftlich umso wirksamer, je schärfer sie als religiöse Inhalte profiliert sind. Anders gesagt: Je unverbrüchlicher religiöse Akteure ihre Wahrheiten glauben, umso wirksamer bestimmen diese ihr Verhalten. Zudem eröffnet die Verabreitung gesellschaftlicher Lagen vermittels religiöser Logik neue Deutungsmöglichkeiten und Perspektiven für die gesellschaftlichen Vorgänge. Sie ermöglicht also ein distinkt religiöses Handeln im Blick auf allgemein geteilte gesellschaftliche Bedingungen also genau das, was leicht als »eigengesetzliche« religiöse Praxis erscheint.

Bei NPERG interpretiert die Anwendung des Exorzismus-Schemas gesellschaftliche Gegensätze in einer Weise, die Gegner satanisiert und die Machtfrage symbolisch klärt. Dadurch erhält das gesellschaftliche Handeln der Akteure Durchschlagskraft und wird mit Siegesgewissheit unterfüttert - eine klassisch fundamentalistische Strategie der Absolutsetzung bzw. Selbst-Sakralisierung. ${ }^{53}$ Des Weiteren werden die Laufbahnperspektiven der Akteure erweitert, indem das Ziel der ökonomischen Prosperität um die politische Perspektive eines gesellschaftlichen Wandels durch eine theokratische Restauration ergänzt wird. Die religiöse Transformation der Perspektive ergänzt so ökonomische Prätention durch eine neue Perspektive politischen Handelns. Im Blick auf die Problematik gesellschaftlicher Differenzierung - und die damit zusammenhängende objektive Bestreitung religiöser Universalkompetenz - verbindet sich eine Totalisierungsstrategie mit Offenheit für funktionale Differenzierung, indem der Geist (zusammen mit vielfältigen Dämonen) als vielgestaltiger Universaloperator in verschiedensten Praxisfeldern den unterschiedlichsten Rollenerwartungen entsprechend eingesetzt wird. Gemäß der postmillenaristischen Geschichtskonzeption der NPERG konstruieren alle diese Strategien einen innergeschichtlichen, aber dennoch absoluten Raum religiöser Andersartigkeit, in dem die gesellschaftlichen Verhältnisse neu definiert werden.

PERG/U konstruiert einen solchen Raum gegen die Geschichte als ganze. Bei fehlenden sozialen Handlungsmöglichkeiten wird geschichtliches, d. h. die Gesellschaft gestaltendes, Handeln als Alternative prinzipiell ausgeschlossen und die Entrückung ins Jenseits als religiöse Realität konstruiert. Und eben daraus resultieren Selbstausgrenzung und Rückzug auf interne Gruppensolidarität als reale diesseitige Überlebensstrategie. Damit wird die kirchliche Welt zu einer Gegenwelt, in der die Werte der Gesellschaft umgekehrt werden: das ohnehin Unerreichbare z. B. Schmuck, politischer Einfluss etc. - erscheint als Unwert gegenüber dem wahren Wert der asketischen Heiligkeit.

In beiden Fällen besteht die spezifisch religiöse Operation darin, dass eine »nicht weltliche Welt« konstruiert wird: ein Raum, der »weltlichen « Logiken prinzipiell nicht zugänglich ist. In diesem Raum entsteht eine Logik, unter der die Akteure der Welt begegnen können - und zwar positionsspezifisch. Mit anderen Worten, die spezifisch religiöse Funktion religiöser Symbolik liegt in der Konstruktion einer Transzendenz, die der gesellschaftlichen Position entspricht.

53 H. Schäfer, »Religious fundamentalism...«. 


\section{Fazit: Positionen und Dispositionen}

Wie verhalten sich religiöse und gesellschaftliche Praxis zueinander? Sind es die gesellschaftlichen Positionen der Akteure, die religiöse Bewegungen wie die Pfingstbewegung in unterschiedliche Habitusformationen ausdifferenzieren?

Unsere Untersuchung »der « Pfingstbewegung in Guatemala hat gezeigt, dass gesellschaftliche Positionen religiöse Dispositionen in hohem Maße strukturieren. Das ist, wenn man so will, die andere Seite der >Diesseitigkeit< (Weber) religiöser Logik: Selbst wenn sich Akteure - wie die traditionellen Pfingstler - apokalyptisch radikal am Jenseits orientieren, ist ihre Logik des Wahrnehmens, Urteilens und Handelns objektiv strategisch am Interesse des Überlebens in aussichtsloser Lage ausgerichtet. Und wenn für kapitalstarke Akteure - wie die Neopfingstler - die gesellschaftliche Machtfrage auf dem Spiel steht, dann dient ihnen charismatische Religion zu subjektiv strategischer Machtpolitik.

Das religiöse Feld hat unbezweifelbar eine Eigendynamik, die von seinen unterschiedlichen Positionen in der objektiven Konkurrenz um religiöses Kapital und das Monopol im Feld abhängt. Aber selbst diese Eigenlogik ist - insbesondere in Konjunkturen raschen sozialen Wandels - keineswegs unabhängig von den gesellschaftlichen Strukturen und Prozessen.

Der Schwerpunkt des vorliegenden Aufsatzes liegt auf den objektiven Relationen zwischen religiöser Praxis und gesellschaftlichen »Funktionssystemen«. Deren Modellierung mittels des Theorems der Entsprechungen zwischen - strikt akteursbezogener - Logik der Praxis und praktischer Logik hat sich als fruchtbar erwiesen. Sie hat generelle (1. und 2.) sowie auch sehr spezifische (3.1 und 3.2) Entsprechungen zu beschreiben erlaubt. Unterschiedliche strukturanalytische Aspekte (Klassenlage, Modernisierung/Rationalisierung, Differenzierung etc.) wurden im Konzept der Logik der Praxis der Akteure berücksichtigt und konnten mit den Ergebnissen einer Analyse der praktischen Logik der Akteure vermittelt werden. Ebenso war es durch den Ansatz bei Bourdieu möglich, religionssoziologische Impulse marxscher (Einbindung der Religion in gesellschaftliche Konflikte), durkheimscher (Isomorphie-Annahme) und weberscher (Sinn, Interessen) Provenienz zu berücksichtigen.

Unsere Ausgangsfragen, ob und wie gesellschaftliche und religiöse Praxis vermittelt sind und wie sich das religiöse Feld zur Machtverteilung im gesellschaftlichen Raum verhält, können damit zwar nicht als umfassend beantwortet gelten. Aber mindestens konnte eine Möglichkeit durchgespielt werden, Positionen und Dispositionen von religiösen Akteuren analytisch in Beziehung zu setzen und einige Formen der Vermittlung aufzuzeigen. Die praktische Logik der Religion erwies sich als höchst kreative Kraft der Entwicklung >diesseitiger < Strategien. Es hat sich wieder einmal gezeigt, dass - wie Durkheim feststellte ${ }^{54}$ - noch die seltsamsten Riten und fremdesten Mythen auf Bedürfnisse des individuellen und sozialen Lebens zurückgehen und hinter jedem Symbol eine soziale Wirklichkeit liegt, die ihm überhaupt erst Bedeutung und Sinn gibt.

54 Vgl. E. Durkheim, Die elementaren Formen..., 19. 
Im Blick auf die Pfingstbewegung lässt sich abschließend Folgendes sagen: Die »Laborsituation « gesellschaftlicher Polarisierung im Bürgerkrieg Guatemalas der achtziger Jahre hat es erlaubt, exemplarisch zwei diametral entgegengesetzte Positionen in der Pfingstbewegung herauszuarbeiten: Welt-erobernde Charismatik und Welt-fliehende Apokalyptik. Schon damals hat es in Guatemala nicht nur diese beiden Extreme gegeben. Und mit einer geringeren Polarisierung einer Gesellschaft und diversifizierten Handlungschancen von Akteuren kann man eine breite Differenzierung von Habitusformationen in einer religiösen Bewegung annehmen (was ja heute empirisch in Lateinamerikas Pfingstbewegung überdeutlich ist). Meiner Ansicht nach kann man aber auch dann immer signifikante Zusammenhänge zwischen gesellschaftlicher Position der jeweiligen Akteure und ihrer religiösen Praxis aufzeigen. Damit kann das hier vorgestellte Beschreibungsmodell gerade bei starker Differenzierung von Nutzen sein. 